\title{
Geometry of the Space of Triangulations of a Compact Manifold
}

\author{
A. Nabutovsky \\ Courant Institute of Mathematical Sciences, 251 Mercer St, New York University, New York, NY \\ 10012, USA E-mail: alex@math toronto.edu
}

Received: 6 October 1994/Accepted: 1 February 1996

\begin{abstract}
In this paper we study the space $T_{M}$ of triangulations of an arbitrary compact manifold $M$ of dimension greater than or equal to four. This space can be endowed with the metric defined as the minimal number of bistellar operations required to transform one of two considered triangulations into the other. Recently, this space became an object of study in Quantum Gravity because it can be regarded as a "toy" discrete model of the space of Riemannian structures on $M$.

Our main result can be informally explained as follows: Let $M$ be either any compact manifold of dimension greater than four or any compact four-dimensional manifold from a certain class described in the paper. We prove that for a certain constant $C>1$ depending only on the dimension of $M$ and for all sufficiently large $N$ the subset $T_{M}(N)$ of $T_{M}$ formed by all triangulations of $M$ with $\leqq N$ simplices can be represented as the union of at least $\left[C^{N}\right]$ disjoint non-empty subsets such that any two of these subsets are "very far" from each other in the metric of $T_{M}$. As a corollary, we show that for any functional from a very wide class of functionals on $T_{M}$ the number of its "deep" local minima in $T_{M}(N)$ grows at least exponentially with $N$, when $N \rightarrow \infty$.
\end{abstract}

\section{Introduction}

Let $M$ be a compact PL-manifold, $T_{M}$ be the (discrete) set of all triangulations of $M$. (By a "triangulation of $M$ " we mean in this paper a simplicial complex such that its space is PL-homeomorphic to $M$. We do not distinguish between simplicially isomorphic triangulations and regard them as identical.) There are many ways to introduce a natural metric on $T_{M}$. For example, the results of Pachner imply that any triangulation of $M$ can be transformed into any other triangulation of $M$ by a finite sequence of bistellar operations ([P1,P2]). (A bistellar operation can be defined as follows. Let $n$ denote the dimension of $M$. Consider a subcomplex $K$

Current Address: Department of Mathematics, University of Toronto, Toronto, Ontario, Canada, M5S 3G3

This work was partially supported by the New York University Research Challenge Fund Grant, by Grant ARO-DAAL-03-92-G-0143 and by NSERC Grant OGP0155879 
of the considered triangulation of $M$ with the following property: $K$ is simplicially isomorphic to a subcomplex of the boundary of the standard $(n+1)$-dimensional simplex $\Delta^{n+1}$ which consists of $k n$-dimensional simplices and all their faces, where $k \in\{1,2, ., n+1\}$ A bistellar operation consists of removing this subcomplex $K$ from the triangulation and replacing it by the complementary subcomplex of the boundary of $4^{n+1}$ containing all remaining $n$-dimensional simplices and their faces ) Therefore one can define the distance between two triangulations of $M$ as the minimal number of bistellar operations required to transform one of them into the other Thus, $T_{M}$ becomes a metric space. (Alternatively, one can use, for example, the Alexander simple transformations ([A]) instead of bistellar operations to introduce a metric on $T_{M}$ ) Let $T_{M}(N)$ denote the set of all triangulations of $M$ with not more than $N$ simplices. In this paper we prove several quantitative results describing "bad" properties of geometry of subsets $T_{M}(N)$ of the metric space $T_{M}$ for large $N$. For example, we prove that for any $n \geqq 5$ there exists a constant $C(n)>1$ such that for any compact $n$-dimensional manifold $M$ and any Turing computable function $\theta(N)($ say, $[\exp \exp \exp (N)]$ ( $N$ times)) for all sufficiently large $N$ there exist $\left[C^{N}(n)\right]$ triangulations $T_{1}, \quad, T_{\left[C^{N}(n)\right]}$ of $M$ with $\leqq N$ simplices such that $\operatorname{dist}\left(T_{l}, T_{j}\right)>\theta(N)$ if $i \neq j$ (Corollary 1.2). (However, this result is false without the assumption about Turing computability of $\theta$. Also, we would like to stress that $C(n)$ does not depend on $\theta$, although the minimal $N$ for which such $\left[C^{N}(n)\right]$ triangulations exist depends on $\theta$.) Informally, this result implies that for large $N T_{M}(N)$ is a union of at least $\left[C^{N}(n)\right]$ disjoint non-empty subsets which are "very far" from each other This result can be generalized for a very wide class of metrics on $T_{M}$ (see Corollary 13 ). Some further generalizations of this result (including a generalization for a class of four-dimensional manifolds) and related results are described in Sect 1 In particular, we consider a class of discrete variational problems on $T_{M}$ and show that these problems have "many" solutions (Theorem 1.5). Our interest in geometry of $T_{M}$ and in discrete variational problems on this space is partially motivated by the fact that it can be regarded as a "toy" discrete model of the space of Riemannian structures of a fixed volume on $M$ (for smooth $M$ ) (The idea is that given a triangulation one can define a singular metric on the manifold assuming that all one-dimensional simplices are of the same length. This informal approach to discretization of the space of Riemannian structures is well-known in String Theory and Quantum Gravity (cf $[\mathrm{AM}, \mathrm{AJK}, \mathrm{J}]$ ), where it is called the "dynamical triangulation approach" This approach is a simplified version of the Regge calculus (see [CMS] and references there) Theorem 1.5 implies that the action functionals considered in $[\mathrm{AM}]$ or $[\mathrm{AJK}]$ will have infinitely many "deep" local minima in $T_{M}$ and the number of these minima among triangulations with $\leqq N$ simplices exponentially grows with $N$ ) Thus, it is not surprising that the results of the present paper have differential-geometric analogues For example, a differential-geometric analogue of Corollary 1.2 is Conjecture 1.6 in Sect 1 This conjecture describes the geometry of the sets of Riemannian structures of volume one and injectivity radius greater than $\varepsilon$ on a fixed compact manifold of dimension greater than three, when $\varepsilon \rightarrow 0$, and can be approached using the methods of the present paper (see [N2, N5]).

The method of investigation of $T_{M}$ used in this paper is a quantitative version of the method used in $[\mathrm{ABB}$ and $\mathrm{NBA}]$ (and also in a different context in [N0, N1 and N4], see also [Grl, p 212]) and based on S Novikov's theorem establishing the algorithmic unrecognizability of the sphere $S^{n}$ for any $n \geqq 5$ This $S$ Novikov theorem is proven using the algorithmic unsolvability of the triviality problem for finitely presented groups which, in turn, follows from the algorithmic unsolvability 
of the halting problem for Turing machines. The crucial technical idea of the present paper is to replace the unsolvability of the halting problem for Turing machines in the foundation of this approach by Barzdin's result providing an exponential lower bound for the time-bounded Kolmogorov complexity of the halting problem for a certain Turing machine. It turns out that this modification leads to much stronger results. The time-bounded Kolmogorov complexity of an unsolvable decision problem is, roughly speaking, the minimal amount of an auxiliary oracle information required to solve the problem for all instances of size $\leqq N$ in a time bounded by a prescribed computable function of $N$. (This amount is regarded as a function of $N$. We assume that there is a natural notion of size of instances of the problem. The auxiliary information is represented as a binary sequence. Its amount is just the length of the sequence. Informally, one can imagine that the decision problem must be solved using a program, say in FORTRAN or PASCAL, where only integer data type is allowed. This program is supposed to work as follows. For every $N$ it receives as input data a certain amount of arbitrary auxilliary oracle information which can be, for example, a partial list of answers for the decision problem, etc. Then the program must be able to find the answer for the decision problem for any given instance of size $\leqq N$. Moreover, it must be able to complete this computation in a time not exceeding a prescribed Turing computable function of $N$. A formal definition of time-bounded Kolmogorov complexity will be given in Sect. 3.) Barzdin ([B]) has shown that there exists a recursively enumerable set $I \subset \mathbb{N}$ with the following property. Consider the decision problem $P$ "Whether or not a given positive integer number is in $I$ ?" Let us regard the length $L$ of the binary expansion of an integer number $N$ (i.e. $\left[\log _{2}(N)\right]+1$ ) as its size. Then for any computable (i.e. recursive) function $t(L)$ for all sufficiently large $L$ the Kolmogorov complexity of the decision problem $P$ with time resources bounded by $t(L)$ is at least $c_{t} 2^{L}$ for a certain positive constant $c_{t}$ (depending on $t$ but not on $N)$. (That is, one requires at least $c_{t} 2^{L}-$ const bits of oracle information to be able to solve the problem $P$ for all integer numbers of length $\leqq L$ in time not exceeding $t(L))$. This result of Barzdin can be used to show that for any Turing computable function $t(N)$ for all sufficiently large $N$ the Kolmogorov complexity of the problem of recognition of $S^{n}$ (or any other compact $n$-dimensional manifold $M$ ) for $n \geqq 5$ among triangulations with $\leqq N$ simplices in time bounded by $t(N)$ is not less than const $(n)^{N} /$ const $_{t}$, where $\operatorname{const}(n)>1$ is a constant depending only on $n$ and const $t>0$ depends only the time bound $t$ and on the manifold $M$. To prove this lower bound one must find an "economical" effective reduction of the halting problem for Turing machines to the recognition problem for $M$. This reduction is provided by Theorem 2.1. Although its proof essentially follows the known proofs of the unsolvability of the triviality problem for finitely presented groups and of the S. Novikov theorem, a serious difficulty arises due to the fact that S. Novikov uses a technique from homology algebra making one of his constructions not very effective. To overcome this difficulty we provide an explicit and economical (albeit tedious) construction of a homology sphere such that its fundamental group is the universal central extension of a prescribed perfect finitely presented group.

Starting from this point our method works as follows. Consider some characteristic of geometric complexity of $T_{M}(N) \subset T_{M}$ (for example, the minimal number of metric balls of radius $\theta(N)$ with centers in $T_{M}(N)$ required to cover $T_{M}(N)$ ). Then we try to prove an upper bound for the Kolmogorov complexity of the problem of recognition of $M$ with a specific Turing computable time limit in terms of the chosen geometric complexity exhibiting a specific algorithm "quickly" recognizing 
$M$ using a specific oracle information. For example, we can request from an oracle a description of a representative from every ball in a collection of balls of radius $\theta(N)$ with centers in $T_{M}(N)$ covering $T_{M}(N)$. It is not difficult to see (see the proof of Lemma 3.2) that one can "quickly" recognize $M$ among triangulations with $\leqq N$ simplices using this collection of representatives. Now the juxtaposition of this upper bound for the Kolmogorov complexity and of the lower bound following from the Barzdin result implies a lower bound for the minimal number of balls of radius $\theta(N)$ required to cover $T_{M}(N)$ (or, more generally, for a considered characteristic of geometric complexity of $\left.T_{M}(N) \subset T_{M}\right)$. A detailed description of this method is given in Sect. 3.

This technique based on Theorem 2.1 and on the usage of time-bounded Kolmogorov complexity can be applied to study geometry of various moduli spaces arising in differential geometry. I discuss some potential applications of this technique at the end of Sect. 1 ( see also [N2, N3, N5]).

\section{Main Results}

Using the unsolvability of the triviality problem for finitely presented groups (proven independently by Adyan and M. Rabin, cf. [Mi]) Markov has constructed a triangulation $T_{0}$ of some compact 4-dimensional manifold $M_{0}$ such that there is no algorithm recognizing whether or not a given triangulation $T$ of a $P L$-manifold is combinatorially equivalent to $T_{0}$ (i.e. the space of $T_{0}$ is $P L$-homeomorphic with $M_{0}$ ). Several years afterwards S P Novikov proved that for any $n \geqq 5$ the sphere $S^{n}$ is algorithmically unrecognizable (in particular, in the sense above). His proof was published in 1974 as ch.10 of the paper [VKF]. His result easily implies that any compact $P L$-manifold of dimension $\geqq 5$ is algorithmically unrecognizable (It is not known whether or not $S^{4}$ is algorithmically unrecognizable ) In Sect. 2 we prove a quantitative version of this result of Novikov. Namely, we demonstrate the existence of an algorithm which for any given $n \geqq 5$, any given compact PLmanifold $M^{n}$, any recursively enumerable set $I \subset \mathbb{N}$, presented as the halting set of a given Turing machine $T$, and any natural number $k$ constructs a triangulation of a compact PL-manifold $M_{k}^{n}$ such that: 1) $M_{k}^{n}$ is PL-homeomorphic to $M^{n}$ if and only if $k \in I$, and 2) The number of $n$-dimensional simplices in the constructed triangulation does not exceed const $(n, T) \ln k+\left|M^{n}\right|$, where const $(n, T)$ does not depend on neither $k$ nor $M^{n}$ and $\left|M^{n}\right|$ denotes the number of the $n$-dimensional simplices in the given triangulation of $M^{n}$ This result then is applied to a study of geometry of the space of all triangulations of $M^{n}$ as follows.

Let $M$ be an algorithmically unrecognizable compact PL-manifold (for example, $M$ can be any compact manifold of dimension greater than four). Then, as it is known, there is no algorithm which constructs for any given $N$ the list of all triangulations of $M$ with $\leqq N$ simplices of all dimensions (see [ABB and NBA] for this and related results). (Indeed, assume the opposite. Then it would be possible to recognize $M$ using the following algorithm. Let $T$ be a given triangulation with $N(T)$ simplices Construct all triangulations of $M$ with $\leqq N(T)$ simplices. Now we can compare $T$ with all the triangulations on this list checking every time whether or not $T$ is simplicially isomorphic to the considered triangulation Since it is clearly possible to check whether or not two simplicial complexes are simplicially isomorphic (cf $[\mathrm{ABB}]$, Proposition 2.16), we have an algorithm recognizing $M$. This yields the desired contradiction with the unrecognizability of $M$.) 
Having this in mind, it is natural to consider algorithms providing for any given $N$ and any given triangulation $T_{0}$ of a compact $P L$-manifold $M$ with $\leqq N$ simplices a partial list of triangulations of $M$ with $\leqq N$ simplices and completing this work in a time bounded by a Turing computable function of $N$. Formally speaking, the term algorithm" means in this context a partial recursive function from the product of $\mathbb{N}$ with the set of finite abstract simplicial complexes (regarded as sets of subsets of the set $\{1, \ldots, v\}$ of vertices of the simplicial complex) of a fixed dimension $n=\operatorname{dim}(M)$ to the set of finite sets of finite abstract simplicial complexes. The domain of this function must include all pairs $(N, T)$, where $T$ is a triangulation of $M$ with $\leqq N$ simplices, and, moreover, this function must be Turing computable in a time bounded by a recursive function of $N$. The requirement of Turing computability of this function in a recursive time is not automatically satisfied since, in general, the set of all simplicial complexes triangulating $M$ is not a recursive subset of the set of all finite abstract simplicial complexes. However, this requirement will be automatically satisfied if the algorithm is defined not only for all triangulations of $M$ but for all finite simplicial complexes of the dimension $\operatorname{dim}(M)$ with $\leqq N$ simplices (or even just for all finite simplicial complexes with $\leqq N$ simplices triangulating pseudomanifolds). This will be the case for all specific algorithms, considered in this paper.

Let $A$ be such an algorithm. For any $N$ we introduce the relation $\geqq_{A}$ on the set $T_{M}(N)$ of all triangulations of $M$ with $\leqq N$ simplices as follows: $T_{1} \geqq_{A} T_{2}$ if and only if the triangulation $T_{1}$ of $M$ is on the list of triangulations of $M$ obtained by the application of $A$ to $T_{2}$ and $N$. The reflexive transitive closure of this relation denoted by $\geqq^{A}$ is a quasi-order on $T_{M}(N)$. As usual, we write $T_{1}={ }^{A} T_{2}$ if and only if $T_{1} \geqq^{A} T_{2}$ and $T_{2} \geqq^{A} T_{1}$. The relation $={ }^{A}$ is an equivalence relation on $T_{M}(N)$. The set $T_{A, M}(N)$ of equivalence classes of this relation is a poset with the order $\geqq^{A}$ inherited from $T_{M}(N)$. We conjecture that the number $m_{A, M}(N)$ of minimal elements of $T_{A, M}(N)$ grows at least exponentially with $N$ for any compact manifold $M$ of dimension at least four and for any algorithm $A$. The main result of this paper is that this conjecture holds in the case when the dimension of $M$ is greater than four and also for a class of four-dimensional manifolds:

\section{Theorem 1.1.}

A. For any $n \geqq 5$ there exists $C(n)>1$ such that for any compact $n$ dimensional PL-manifold $M$ and any algorithm $A$ there exists $N_{0}(A, M)$ with the following property: For any $N \geqq N_{0}(A, M)$,

$$
m_{A, M}(N)>C^{N}(n) .
$$

B. There exists a constant $C(4)>1$ and an integer number $k$ such that for any algorithm $A$ and any compact four-dimensional PL-manifold $M_{0}^{4}$ there exists $N_{0}\left(A, M_{0}^{4}\right)$ with the following property: Let $M$ be the connected sum of $M_{0}^{4}$ and $k$ copies of $S^{2} \times S^{2}$. Then for any $N \geqq N_{0}\left(A, M_{0}^{4}\right)$,

$$
m_{A, M}(N)>C^{N}(4) \text {. }
$$

Remark 1 We define $T_{M}(N)$ as the set of triangulations of $M$ with $\leqq N$ simplices of all dimensions. Alternatively we could define it as the set of triangulations of $M$ with $\leqq N$ simplices of the maximal dimension and then define the poset $T_{A, M}(N)$ exactly as it was defined. Theorem 1.1 will remain true in this case with virtually the same proof. 
Remark 2 Note that many of the results of $[\mathrm{ABB}]$ can be interpreted as the statement that for certain algorithms $A$ and for any compact PL-manifold of dimension $\geqq 5$ for all sufficiently large $N m_{A, M}(N)>1$

The proof of Theorem 11 is based on the analysis of time-bounded Kolmogorov complexity of the decision problem, "Is a given compact PL-manifold PL-homeomorphic to M?"

Consider an algorithm $A$ satisfying the following very mild restriction. There exists an algorithm $A^{-1}$ constructing for a given $N$ and a given triangulation $T \in T_{M}(N)$ the list of all triangulations $T_{1} \in T_{M}(N)$ such that $T$ is on the list $A\left(N, T_{1}\right)$ (All algorithms considered in this paper satisfy this condition. This condition holds, for example, when the algorithm $A$ can be applied to arbitrary triangulations (not just triangulations of $M$ ) producing triangulations of the same polyhedron, or when $\geqq_{A}$ is an equivalence relation. In this last case one can take $A^{-1}=A$.) Consider the algorithm $\bar{A}$ producing for given $T$ and $N$ the union of the lists $A(N, T)$ and $A^{-1}(N, T)$. (Note that if $\geqq_{A}$ is symmetric, then $\bar{A}=A$.) It is clear that $\geqq^{\bar{A}}$ is an equivalence relation on $T_{M}(N)$, and $T_{M}(N)$ can be represented as the union of $m_{\bar{A}, M}(N)$ disjoint equivalence classes with respect to this relation. We will call these equivalence classes $A$-simple sets (It is clear that there exists an algorithm constructing for a given $N$ and a triangulation $T \in T_{M}(N)$ the $A$-simple set containing $T$ This observation justifies the name $A$-simple sets.) Theorem 1.1 implies that for all sufficiently large $N$ the number of $A$-simple sets in the representation of $T_{M}(N)$ as a union of disjoint non-empty $A$-simple sets is greater than $\left[C^{N}(n)\right]$ Informally speaking, $A$-simple sets (for a fixed algorithm $A$ ) can be regarded and used as an analog of connected components of $T_{M}(N)$ (see Remark 1 after Corollary 1.3 and the proof of Theorem 15$)$ Therefore, it is desirable to know more about general properties of partitions of $T_{M}(N)$ into $A$-simple sets. The fact that one can prove an independent of $A$ asymptotic lower bound on the number of sets in such partitions (provided by Theorem 1.1) encourages me to ask several further questions:

What can be said about the possible distributions of sizes of A-simple sets when $N \rightarrow \infty$ ?

(This question can be compared with the discussion in Sect. $4 B_{1}$ of [Gr2]. The question is of interest also for specific algorithms $A$, for example, for the algorithm $A$ which for given $N$ and $T$ produces all triangulations of $M$ with $\leqq N$ simplices which can be obtained from $T$ by one bistellar operation or, alternatively, by a sequence of, say, $2^{2^{N}}$ bistellar operations.)

How large is the number $L_{A, M}(N)$ of elements in the maximal A-simple set in comparison with the number $t_{M}(N)$ of all triangulations of $M$ with $\leqq N$ simplices? In particular, is it true that $\sup _{A} \lim \sup _{N \rightarrow \infty} \frac{L_{A, M}(N)}{t_{M}(N)}<1$ ?

(In other words we ask how large is a part of the set of all triangulations of $M$ with $\leqq N$ simplices which can be recovered using some fixed algorithm, when $N \rightarrow \infty$ ? This question is of interest also for Quantum Gravity; see the discussion at the end of [NBA].)

Now I am going to describe several specific applications of Theorem $1.1 \mathrm{~A}$ class of the algorithms of the considered kind is based on the usage of elementary moves (or elementary transformations). The most well-known examples of such elementary moves are the Alexander simple transformations of arbitrary order (see the 
definition in [A, p. 299] or in [G, p. 8], where the Alexander simple transformations are called "elementary starrings") and their inverses (called in [G] "elementary weldings"). These operations do not change a combinatorial equivalence class of the simplicial complex. Alexander proved that any two triangulations of a compact $P L$ manifold can be transformed one into another by a finite sequence of the Alexander simple transformations (elementary starrings and weldings) (see [A, Theorem 15.1] and [G, Theorem II.17]). Another such set of elementary moves is called "bistellar operations" or "Pachner's moves" (cf. [P1, P2].) (The definition of the bistellar operations was given in the Introduction above). U. Pachner proved ([P1, P2], see also $[\mathrm{GV}])$ that any two triangulations of the same compact manifold can be connected by a sequence of bistellar operations. Given a set of elementary moves and a recursive (i.e. Turing computable) function $\theta(N)$ (say, $\theta(N)=N^{N^{N}}$ ) one can define an algorithm which will apply all possible chains of $\leqq \theta(N)$ elementary moves. Its output is the list of all triangulations with $\leqq N$ simplices which can be obtained in this way. Denote this algorithm in the case when the set of elementary moves is the set of all bistellar operations for the considered dimension by $A_{\text {bistellar, } \theta}$. The relation $\geqq A_{\text {bistellar, } \theta}$ is a symmetric relation on $T_{M}(N)$. Hence, the resulting poset $T_{A_{\text {bistellar }, \theta}, M}(N)$ will be an antichain and all its elements will be minimal. Being applied to $A_{\text {bistellar, } \theta}$ Theorem $1.1 \mathrm{~A}$ easily implies that:

Corollary 1.2. Let $n$ be greater than four and $C(n)>1$ be the constant defined in Theorem 1.1A. For any compact PL-manifold $M$ of dimension $n$ and for any Turing computable function $\theta$ there exists $N_{0}$ with the following property: For any $N \geqq N_{0}$ there exists triangulations $T_{1}, T_{2}, \ldots, T_{\left[C^{N}(n)\right]}$ of $M$ with not more than $N$ simplices such that there is no sequence of less than $\theta(N)$ bistellar operations transforming one of these triangulations to another of these triangulations.

As it was noted in the introduction one can define a metric on $T_{M}$ as the minimal number of bistellar operations required to transform one of two considered triangulations of $M$ into the other triangulation. Corollary 1.2 can then be regarded as a lower bound for the number of balls of radius $\theta(N) / 2$ required to cover $T_{M}(N)$. This version of Corollary 1.2 can be stated for a very general class of metrics on $T_{M}$ :

Corollary 1.3. Let $M$ be a compact PL-manifold of dimension $n \geqq 5$. Let dist : $T_{M} \times T_{M} \rightarrow \mathbb{R}$ be a metric on $T_{M}$ satisfying the following condition: There exists an algorithm constructing for a given triangulation $T$ of $M$ and given integer numbers $N$ and $K$ the set of all triangulations $S$ of $M$ with $\leqq N$ simplices such that $\operatorname{dist}(S, T) \leqq K$ in a time bounded by a recursive function of $N, K$ and the number of simplices in $T$. Then for any recursive function $\theta$ for all sufficiently large $N$ the minimal number of metric balls of radius $\theta(N)$ in $T_{M}$ required to cover $T_{M}(N)$ is not less than $\left[C^{N}(n)\right]$, where $C(n)>1$ is the constant defined as in Theorem 1.1A.

Indeed, one can immediately prove Corollary 1.3 applying Theorem 1.1 to the algorithm $A_{\text {dist,2 } 2 \theta}$ which for any triangulation from $T_{M}(N)$ finds the intersection of its neighborhood of radius $2 \theta(N)$ (in the metric dist) with $T_{M}(N)$.

Remark 1. One can better understand the geometric meaning of Corollary 1.3

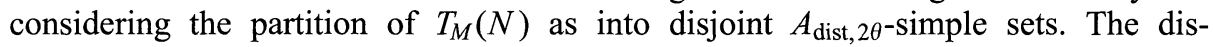
tance between any two triangulations from different $A_{\text {dist, } 2 \theta}$-simple sets will be more than $2 \theta(N)$. Thus, informally one can regard these $A_{\text {dist, } \theta} \theta$-simple sets as 
connected components of $T_{M}(N)$ Corollary 1.3 says that for large $N$ there will be at least $\left[C^{N}(n)\right]$ sets in this partition.

Remark 2 The techniques of [N0] and [ABB] immediately imply the following result explicitly stated (in an equivalent form) in [NBA]: The diameter of $T_{M}(N)$ in the metric considered in Corollary 13 cannot be majorized by any recursive function of $N$ This result is much weaker than Corollary 1.3

Another class of algorithms $A$ has the following property The relation $\geqq^{A}$ defined as above on $T_{M}(N)$ is antisymmetric for any $N$. (Thus, the relation $={ }^{A}$ is trivial, and every equivalence class of $={ }^{A}$ contains only one triangulation.) For some non-trivial algorithms $A$ the minimality of a triangulation with respect to $\geqq^{A}$ has interesting combinatorial interpretations This suggests the following idea For a combinatorial property of interest we can try to find an algorithm $A$ such that the relation $\geqq^{A}$ on the set $T_{M}(N)$ of all triangulations of $M$ with $\leqq N$ simplices is antisymmetric and the minimality of a triangulation with respect to $\geqq^{A}$ is equivalent to the considered combinatorial property For example, if we are interested in triangulations which are not rectilinear subdivisions of another triangulation, then the corresponding algorithm will be the algorithm described in [ABB], finding all rectilinear subdivisions of a given triangulation of $M$ with $\leqq N$ simplices. Thus, Theorem 1.1 will immediately imply the existence of infinitely many triangulations $M$ which are not rectilinear subdivisions of another triangulation for every compact manifold $M$ of dimension $\geqq 5$ (Let us call such triangulations prime. More formally, (a simplicial isomorphism class of) a (finite) simplicial complex $C_{1}$ is called a prime triangulation if there is no simplicial complex $C_{2}$ with the following properties. (a) The number of simplices in $C_{2}$ is less than the number of simplices of $C_{1}$, and (b) There exists geometric realizations of $C_{1}$ and $C_{2}$ in an Euclidean space such that the geometric realization of $C_{1}$ is a rectilinear subdivision of the geometric realization of $C_{2}$.) Actually, it is not difficult to construct explicit examples of prime triangulations even in the case when the dimension of $M$ is equal to three (Such a construction can be found, for example, in [Ca]. See also $[\mathrm{CH}]$ for a discussion of related questions. I would like to thank Prof R. Connelly, who informed me about this construction and pointed out the references ) However, the described approach permits, for example, to prove the existence of a "large" set of prime triangulations such that no two of these triangulations can be transformed one into another by a "not very long" sequence of bistellar operations More precisely, consider for any Turing computable function $\theta$ an algorithm, which for any given triangulation of $M$ produces the union of the list of all triangulations of $M$ with $\leqq N$ simplices which can be obtained by not more than $\theta(N)$ bistellar operations and the list of all rectilinear subdivisions of $M$ with $\leqq N$ simplices. We can apply Theorem $11 \mathrm{~A}$ to this algorithm. As the result we immediately obtain the following corollary:

Corollary 1.4. Let $n$ be greater than four and $C(n)>1$ be the same as in the text of Theorem 11 For any compact $n$-dimensional PL-manifold $M$, for any Turing computable function $\theta$ for all sufficiently large $N$ there exists at least $\left[C^{N}(n)\right]$ prime triangulations of $M$ with $\leqq N$ simplices with the following property No two of these prime triangulations can be transformed one into the other by less than $\theta(N)$ bistellar operations

Observe, that Corollaries 1.2, 14 will remain true for elementary starrings and weldings instead of bistellar operations One just needs to change the set 
of elementary moves used in the algorithm $A_{\text {bistellar, } \theta}$. Theorem 1.1B implies that Corollaries 1.2-1.4 are also true for all compact four-dimensional PL-manifolds representable as the connected sum of a PL-manifold and $k$ copies of $S^{2} \times S^{2}$, where $k$ is the same number as in Theorem 1.1B. Note also, that the expression "for all sufficiently large $N \ldots$ " in the text of Corollaries $1.2,1.4$ has the following meaning: "there exists a number $N_{0}$ depending on $M$ and $\theta$ such that for any $N \geqq N_{0} \ldots$."

We already mentioned in the introduction that $T_{M}$ can be regarded as a discrete analogue of the space of Riemannian structures on $M$ (for smooth manifolds $M$ ). Moreover, it is used as an easier model for study of the space of Riemannian structures in some papers in Quantum Gravity (e.g. [AM, AJK, J]). These facts provide a motivation for a study of variational problems on $T_{M}$. Let $M$ satisfy the conditions of Theorem 1.1A or B. Consider the set $T_{M}$ of all triangulations of $M$ as the metric space with the metric defined as the minimal number of bistellar operations necessary to transform one of the considered triangulations to another. Alternatively, one can use any other set of elementary moves, for example, the Alexander simple transformations or, more generally, any metric dist satisfying conditions of Corollary 1.3. Let $F: T_{M} \rightarrow \mathbb{R}$ be a functional and $\theta$ be a Turing computable function. We will say that a triangulation $T$ of $M$ is a $\theta$-distinctive local minimum of $F$ if $F(T) \leqq F(S)$ for every triangulation $S \in T_{M}$ such that $\operatorname{dist}(S, T) \leqq \theta(|T|)$. (Here $|T|$ denotes the number of simplices in $T$.) Similarly, one can define $\theta$-distinctive local minima and maxima of the restriction of $F$ on the set $T_{M}(N)$. Consider the algorithm $A_{\text {dist, } \theta}$ which finds for any $N$ and $T \in T_{M}(N)$ the intersection of $T_{M}(N)$ with the ball of radius $\theta(N)$ around $T$ (in the dist metric). Consider the decomposition of $T_{M}(N)$ into the union of disjoint $A_{\text {dist, } \theta}$-simple sets. Denote these sets by $D_{1}, \ldots, D_{l}$. By virtue of Theorem 1.1 for all sufficiently large $N l>C^{N}(n)$. Minima of $F$ on sets $D_{i}$ will be $\theta$-distinctive local minima of the restriction of $F$ on $T_{M}(N)$. Indeed, let $T$ be a minimum of $F$ on $D_{i}$ for some $i$. The definition of $D_{i}$ implies that if $\operatorname{dist}(S, T) \leqq \theta(N)$ and $S \in T_{M}(N)$ then $S \in D_{i}$. Hence, in this case $F(S) \geqq F(T)$. Hence for any computable $\theta$ for all sufficiently large $N$ the restriction of $F$ on $T_{M}(N)$ has at least $\left[C^{N}(n)\right] \theta$-distinctive local minima. Applying a similar but more sophisticated argument one can prove the following theorem (the proof will be given at the end of Sect. 3):

Theorem 1.5. For any $n \geqq 4$ there exists a constant $C_{*}(n)>1$ with the following property: Let either $n \geqq 5$ and $M$ be any compact $n$-dimensional manifold, or $n=4$ and $M$ be any compact four-dimensional PL-manifold representable as a connected sum of a compact PL-manifold and $k$ copies of $S^{2} \times S^{2}$, where $k$ is the constant defined in the text of Theorem 1.1B. Assume that $F: T_{M} \rightarrow \mathbb{R}$ satisfies the following condition: There exists an increasing unbounded Turing computable function $\gamma(N) \leqq N$ such that for any sufficiently large $N$, any triangulation $T_{1}$ of $M$ with $\leqq \gamma(N)$ simplices and any triangulation $T_{2}$ of $M$ with $\geqq N$ simplices $F\left(T_{2}\right) \geqq F\left(T_{1}\right)$. Then for any Turing computable function $\theta$ and for all sufficiently large $N$ the number of $\theta$-distinctive local minima of $F\left(\right.$ on $\left.T_{M}\right)$ with $\leqq N$ simplices is not less than $\left[C_{*}(n)^{\gamma(N)}\right]$. In particular, if for some positive constant const and for all sufficiently large $N \gamma(N) \geqq$ const $N$, then there exists a constant $C_{* *}(n)>$ 1 such that for all sufficiently large $N$ the number of $\theta$-distinctive local minima of $F$ is not less than $\left[C_{* *}^{N}(n)\right]$.

Theorem 1.5 is applicable, for example, to the "regularized action" considered in some papers in Quantum Gravity (e.g. [AM], formulae (1) and (9)). It implies that if $M$ satisfies the conditions of Theorem 1.5 , then this action has infinitely many 
"deep" local minima and that the number of these minima among the triangulations of $M$ with $\leqq N$ simplices grows exponentially with $N$

As it was mentioned in the introduction, the proof of Theorem 11 is based on the following idea involving the notion of time-bounded Kolmogorov complexity. Barzdin found a Turing machine $\tau$ such that the time-bounded Kolmogorov complexity of the halting problem for this machine for any prescribed Turing computable time bound $t$ is not less than $c_{t} 2^{L}$ for all sufficiently large $L$, where a positive constant $c_{t}$ depends on the available time resources and $L$ denotes the length of the considered inputs for $\tau$ (These inputs are binary sequences on the tape.) This result of Barzdin and Markov's proof of the algorithmic unrecognizability of certain manifolds imply for compact four-dimensional manifolds satisfying the conditions of Theorem 1 1B that the time-bounded Kolmogorov complexity of the recognition problem is bounded from below by an exponentially growing function of the number of simplices of a given triangulation (Lemma 31 (b)) Also, Theorem 2.1 together with the Barzdin result imply that a similar lower bound for the time-bounded Kolmogorov complexity of the recognition problem is valid for all compact manifolds of dimension greater than four (Lemma 3.1 (a)). On the other hand it is not difficult to see that if one knows a collection of representatives from all minimal elements of $T_{A, M}(N)$ then one can construct all triangulations of $M$ with $\leqq N$ simplices and, thus, recognize $M$ in the class of all simplicial complexes with $\leqq N$ simplices Moreover, this can be done in a time a priori bounded by a computable function of $N$ (depending on $A$ ). This observation implies an upper bound for the time-bounded Kolmogorov complexity of the recognition problem linearly depending on $m_{A, M}(N)$ (Lemma 32 ). The juxtaposition of these two results implies an exponential lower bound for the number $m_{A, M}(N)$ of minimal elements of $T_{A, M}(N)$

Now I would like to describe several potential applications of the methods of this paper in differential geometry As it was noted, the space $T_{M}$ is regarded in some papers on Quantum Gravity as a discrete analogue of the space of Riemannian structures (i.e. isometry classes of Riemannian metrics) on $M$ because one can assign for every triangulation $T$ of $M$ the same length to all one-dimensional simplices of $T$, obtaining in such a manner a singular piecewise flat metric on $M$. We can choose this length such that the volume of $M$ in this metric will be equal to one. If the number of simplices in $T$ is equal to $N$, then the contractability radius of the resulting metric space is not less than $\operatorname{const}(n) \frac{1}{N^{\frac{1}{n}}}$, where $n=\operatorname{dim}(M)$ Thus, informally, for large $N T_{M}(N)$ can be regarded as a discrete analogue of the space of Riemannian structures on $M$ of volume one and of contractability or injectivity radius greater than $\varepsilon$, where $\varepsilon \approx \frac{1}{N^{1 / n}}$ is small. The corresponding differential-geometric analogue of Corollary 1.2 will be the following:

Conjecture 1.6. For any $n \geqq 4$ there exists a constant $C_{1}(n)>1$ with the following property Let $M$ be a compact smooth differentiable manifold of dimension $n$ greater than four Let for any positive $\varepsilon \operatorname{Riem}_{\varepsilon}(M)$ denote the space of Riemannian structures on $M$ of volume one and injectivity radius greater than $\varepsilon$ For any Turing computable function $\theta$ for all sufficiently small positive $\varepsilon$ there exist $\left[C_{1}^{\frac{1}{\varepsilon^{n}}}(n)\right]$ Riemannian structures from $\operatorname{Riem}_{\varepsilon}(M)$ such that no two of them can be connected by a continuous path in the space $\operatorname{Riem}_{1 / \theta([1 / \varepsilon])}(M)$ of Riemannian structures of volume one and injectivity radius greater than $1 / \theta([1 / \varepsilon])$ on $M$

In particular, Conjecture 16 implies that $\operatorname{Riem}_{\varepsilon}(M)$ is disconnected and the number of path connected components grows at least exponentially with $\frac{1}{\varepsilon^{n}}$, when 
$\varepsilon$ goes to zero. Some results in the direction of Conjecture 1.6 are proven in [N2]. I am going to present a proof of Conjecture 1.6 for $n \geqq 5$ in my paper [N5] which is currently in preparation.

In [N1] I proved the following theorem (Theorem 5.1 in [N1], see also Theorems 5.2 and 4.1): Consider the space of compact $C^{1,1}$-smooth hypersurfaces in $\mathbb{R}^{n+1}$, diffeomorphic to the sphere $S^{n}$, where $n$ is any number greater than four. Consider the functional $\kappa$ on this space defined by the formula $\kappa\left(\Sigma^{n}\right)=\operatorname{vol}^{\frac{1}{n}}\left(\Sigma^{n}\right) / i\left(\Sigma^{n}\right)$, where $i\left(\Sigma^{n}\right)$ denotes the injectivity radius of the normal exponential map of a hypersurface $\Sigma^{n}$ in $\mathbb{R}^{n+1}$ (or, informally, the maximal radius of a non-selfintersecting open tube around $S^{n}$ in $\mathbb{R}^{n+1}$ ). Then the set of values of $\kappa$ at its local minima is unbounded. This result was obtained using the algorithmic unrecognizability of $S^{n}, n \geqq 5$. The method of Sect. 3 can be used to obtain a quantitative information about the distribution of values of $\kappa$ at its local minima.

Another possible application of the technique of the present paper (based on an analysis of the time-bounded Kolmogorov complexity of a relevant membership problem) was suggested to me by Prof. M. Gromov. Namely, in [Gr1] Gromov noted that if the fundamental group of a compact Riemannian manifold has an unsolvable word problem then this manifold has infinitely many geometrically distinct contractible closed geodesics. In [Gr2, Sect. 5.C, p. 102-103] he asks how one can estimate the number of contractible closed geodesics of the length $\leqq x$ in terms of the fundamental group $\pi_{1}(M)$. In [N3] I described the relationship between timebounded Kolmogorov complexity of the word problem for the fundamental group of $M$ and the distribution of lengths of contractible closed geodesics on $M$. In particular, if the fundamental group of a compact Riemannian manifold has a sufficiently "logically complicated" word problem, then the number of contractible closed geodesics of length $\leqq x$ grows at least exponentially with $x$.

\section{An Effective Version of the S. Novikov Theorem on Algorithmic Unrecognizability of $S^{n}, n>4$}

The goal of this section is to prove the following theorem:

Theorem 2.1. There exists an algorithm which for any $n \geqq 5$, any compact $P L$-manifold $M$ of dimension $n$ (presented by a triangulation), any given Turing machine $T$ and its input $w$ constructs a triangulation $R_{T}(w)$ of a compact $n$-dimensional manifold $M_{T}(w)$ such that:

(i) $M_{T}(w)$ is $P L$-homeomorphic to $M$ if and only if $T$ eventually halts, when it starts to work on $w$. If $T$ does not halt with input $w$, then the fundamental groups of $M$ and $M_{T}(w)$ are not isomorphic.

(ii) There exists $c_{n}(T)>0$ depending only on $T$ and $n$ (but not on $w$ and $M$ ) such that the number of simplices in $R_{T}(w)$ does not exceed $c_{n}(T)(|w|+1)+|M|$, where $|w|$ is the length of $w$ and $|M|$ is the number of simplices in the given triangulation of $M$.

Proof. The theorem is an effective version of S. Novikov's theorem on algorithmic unrecognizability of the standard sphere $S^{n}, n \geqq 5$ first published as ch. 10 of [VKF] (see also the detailed exposition in [N4]).

Observe that it is sufficient to construct the algorithm only for the case when $M=S^{n}$ (and is presented by the standard triangulation). In order to get the required 
triangulation $R_{T}(w)$ for an arbitrary $M$ we take the connected sum of the given triangulation of $M$ and the triangulation $R_{T}^{S^{n}}(w)$ constructed for the case $M=S^{n}$

To describe the algorithm for $M=S^{n}$ we start from the observation that the proof of the classical Boone-Novikov theorem stating the unsolvability of the triviality problem for finitely presented groups given in [R] provides for any Turing machine $T$ and its input $w$ an explicit finite presentation of a group $G_{T}$ depending only on $T$ and a word $v$ in $G_{T}$ such that (i) $v$ represents the trivial element of $G_{T}$ if and only if $T$ eventually halts when it starts to work on $w$; (ii) the length of $v$ is bounded from above by $c(T)|w|$, where $c(T)>0$ depends only on $T$. We also observe that the proof of the classical Adyan-Rabin theorem stating the unsolvability of the triviality problem for finitely presented groups given in [Mi], pp. 13-14 actually gives for any finite presentation of a group $G$ and a word $v_{0}$ in $G$ an explicit finite presentation of a group $G_{v_{0}}$ such that (i) the number of generators and the number of relations of $G_{v_{0}}$ depend only on $G$ and the maximal length of a relation of $G_{v_{0}}$ is bounded from above by a linear function of the length of $v_{0}$, (ii) $G_{v_{0}}$ is trivial if and only if $v_{0}$ represents the trivial element in $G$. This finite presentation given by Lemma 3.6 of [Mi] can be described as follows: The set of generators of $G_{v_{0}}$ contains all generators of $G x_{1}, ., x_{q}$, ( $q$ denotes here the number of generators of $G$ ), and also three new generators $a, b, c$. The set of relations of $G_{v_{0}}$ includes all relations of $G$. Besides that it includes $q$ relations

$$
\left[c^{3+1} a^{-3-i}, x_{i} b\right] x_{i}=e, \quad i=1, \ldots, q,
$$

and the following three relations.

$$
\begin{gathered}
c^{-1} b\left[c a^{-1}, b\right]=e, \\
{\left[b a^{2} c^{-2} b^{-1}, c\right] c a^{-1}=e,}
\end{gathered}
$$

and

$$
\left[b, a^{3} c^{-3}\right]\left[v_{0}, b\right] b=e,
$$

where for any $x, y \in G[x, y]$ denotes the commutator of $x$ and $y$. (These relations are equivalent to the formulae (1)-(4) on p. 14 of [Mi].) Observe that only the last of these relations depends on $v_{0}$ Note, that it is clear from this finite presentation of $G_{v_{0}}$ that $G_{v_{0}}$ is perfect, i e that $H_{1}\left(G_{v_{0}}\right)=\{0\}$. Let us apply this construction to the group $G(T)$ and the word $v$ (depending on the given input $w$ ) discussed above. Let us denote the resulting group by $G_{T w}$. $G_{T w}$ will be trivial if and only if $T$ eventually halts, when it starts to work on the input $w$ Below we will need a slightly different finite presentation of $G_{T w}$. Namely, for every generator $g$ of $G_{T w}$. we add a new generator $\bar{g}$ and a relation $g \bar{g}=e$ Now the inverse of every generator of $G_{T w}$, will be equal to another generator of $G_{T w}$. Also, for convenience we prefer at this stage to redenote all generators of $G_{T w}$ by $f_{1}, ., f_{m}$, where $m$ is the number of generators in the finite presentation described above.

Another construction we need is a part of S Novikov's proof For every finite presentation of a perfect group $G$ by generators $f_{i}, i \in\{1,, m\}$ and relators $r_{,}, j \in\{1, ., p\}$ he considers a group $\bar{G}$ defined as follows $\bar{G}$ is generated by the same set of generators $f_{i}$ Its set of relators includes all commutators $\left[f_{1}, r_{l}\right], i \in$ $\{1, \quad, m\}, j \in\{1, \quad, p\}$ and besides that the elements $\tilde{g}_{i}, i \in\{1, \quad, m\}$ defined for every $i$ as follows Since $G$ is perfect (and, thus, coincides with $[G, G]$ ) every generator $f_{i}$ can be represented as a product of an element $\tilde{g}_{l}=\prod_{h} r_{j_{k l}}^{n_{k l}}$ and an element $c_{i} \in[F, F]$, where $F$ is the free group generated by $f_{i}$ It is not difficult 
to see that this group is perfect. Novikov notes that one can prove that $H_{2}(\bar{G})$ is trivial using homological algebra and shows that the kernel of the obvious surjective homomorphism $\bar{G} \rightarrow G$ is isomorphic to $H_{2}(G)$. (Both these statements are consequences of the fact that $\bar{G}$ is the universal central extension of $G$; the proofs can be found in [N4]. We will not need these facts in our proof.) The second statement implies that $\bar{G}$ is trivial if and only if $G$ is trivial. Hence, applying this construction to $G_{T w}$ we obtain a finite presentation of a perfect group $\bar{G}_{T w}$ such that $H_{2}\left(\bar{G}_{T w}\right)$ is trivial, and $\bar{G}_{T w}$ is trivial if and only if $T$ eventually halts when it starts to work on $w$. Moreover, the number of generators and the number of relations of $\bar{G}_{T w}$ do not depend on $w$, and the maximal length of a relator is bounded from above by const $(|w|+1)$. Furthermore, the words $c_{i}$ introduced in the definition of $\tilde{g}_{i}$ can be represented as the product of not more than const commutators of words in $F$ of length bounded from above by a linear function of $|w|$. These facts can be easily seen from the finite presentation of $G_{T w}$ described above. Now, one performs the Dehn construction to build a compact $n$-dimensional manifold $M_{T w}$ such that its fundamental group is $\bar{G}_{T w}$, its second and $(n-2)$-th homology groups are free abelian and homology groups $H_{3}\left(M_{T w}\right), \ldots, H_{n-3}\left(M_{T w}\right)$ are trivial. To construct this manifold one first takes the connected sum of several copies of $S^{1} \times S^{n-1}$. Their number must be equal to the number of generators of $G_{T w}$. Then one realizes relators of $\bar{G}_{T w}$ by embedded circles and performs the surgeries killing these generators (i.e. one deletes small tubular neighborhoods of these circles which are PL-homeomorphic to $S^{1} \times D^{n-1}$ and attaches instead $S^{n-2} \times D^{2}$ ). The resulting manifold will have the required properties. Its second homology group will be generated by the two-dimensional chains corresponding to the axes of the attached 2-handles corresponding to the relators $\left[f_{i}, r_{j}\right]$ in the presentation of $\bar{G}_{T w}$ described above. The resulting manifold can be triangulated using not more than const $(T)(|w|+1)$ simplices of dimension $n$ for some constant const $(T)$.

A classical result of $M$. Kervaire $([\mathrm{K}])$ is that if a finitely presented group $G$ is perfect and $H_{2}(G)$ is trivial, then for every $n \geqq 5, G$ can be realized as a fundamental group of a smooth $n$-dimensional homology sphere. Kervaire's construction (for $G_{T w}$ ) is a part of Novikov's proof. Indeed, by virtue of H. Hopf's theorem $([\mathrm{H}])$ the condition $H_{2}\left(\bar{G}_{T w}\right)=0$ implies that the Hurewicz homomorphism $\pi_{2}\left(M_{T w}\right) \rightarrow H_{2}\left(M_{T w}\right)$ is surjective. Thus, all generators of $H_{2}\left(M_{T_{w}}\right)$ can be represented by PL continuous maps $S^{2} \rightarrow M_{T w}$. These maps can be then approximated by embeddings of $S^{2}$ to $M_{T w}$. Note that small neighborhoods of these embedded 2-dimensional spheres will be PL-homeomorphic to $S^{2} \times D^{n-2}$. A priori knowledge that these spheres exist enables one to find them by a trial and error algorithm. (However, a priori we have no upper bound for the number of simplices required to represent these spheres.) Then one performs surgeries killing these elements of $\pi_{2}\left(M_{T w}\right)$. The result will be a homology sphere $S_{T w}$ with the fundamental group $\bar{G}_{T w}$. Now the application of S. Smale's h-cobordism theorem completes the proof of Novikov's theorem.

An analysis of the described construction implies that Theorem 2.1 would follow from the fact that there exist embedded 2-dimensional spheres realizing the generators of $H_{2}\left(M_{T w}\right)$ and made of not more than $\operatorname{const}_{1}(T)(|w|+1)$ twodimensional simplices in a triangulation of $M_{T w}$ with not more than const $_{2}(T)(|w|+$ 1) simplices.

We are going to prove Theorem 2.1 in a slightly different manner. First, we need a different finite presentation of $\bar{G}_{T w}$. We start from the finite presentation of $\bar{G}_{T w}$ described above. We will call the generators and relators in this finite presentation old 
generators and relators. Let $v \in G(T)$ be the word depending on $w$ and used in the construction of $G_{T_{w}}$. Let $v=\prod_{l=1}^{s} f_{i_{l}}^{\varepsilon_{l}}$, where $s=\operatorname{length}(v), f_{l_{l}}$ are some generators of $G(T)$ (and hence of $G_{T w}$ and $\bar{G}_{T w}$ ) and $\varepsilon_{l}=1$ or -1 Now we introduce new generators $v_{2}, \ldots, v_{l}$ and new relations $v_{2}=f_{l_{1}}^{\varepsilon_{1}} f_{l_{2}}^{\varepsilon_{2}}, v_{l+1}=v_{l} f_{l_{l}}^{\varepsilon_{l}}, l \in\{2, \ldots, s-1\}$ of $\bar{G}_{T w}$. Then we change old relators of the type $[r, f]$, where $r$ is the last relator (involving $[v, b]$ ) of the finite presentation of $G_{T w}$, described above substituting $v_{s}$ for $v$ We also change the old relators of the form $\tilde{g}_{1}$, replacing words $v$ and $v^{-1}$ entering $\tilde{g}_{i}$ by $v_{s}$ and $v_{s}^{-1}$. (The words $v$ and $v^{-1}$ can enter $\tilde{g}_{i}$ because $\tilde{g}_{i}$ are products of relators of $G_{T w}$, and the last relator in the finite presentation of $G_{T w}$ written above involves $v$.) At last, we add $(s-1) p$ new relators of the form $\left[r, v_{i}\right]$, where $r$ runs over the set of relators of $G_{T w}, v_{i}$ runs over the set $\left\{v_{2}, ., v_{s}\right\}$ of all new generators of $\bar{G}_{T w}$, and $p$ denotes the number of relations in the finite presentation of $G_{T w}$ considered above (These new relations are consequences of old relations of $\bar{G}_{T w}$ of the form $[r, f]=e$, where $f$ are old generators. This can be easily proven using the identity $[u, x y]=[u, x][u, y][[y, u], x]$, valid for any elements $u, x, y$ of any group.) It is not difficult to see that we got another finite presentation of the same group $\bar{G}_{T w}$. The numbers of generators and relators of this finite presentation are bounded from above by const $(|w|+1)$ and the length of every relator is bounded by a constant (not depending on $|w|$ ). Note that every generator $f$ of $G_{T w}$ is representable as a product of an element $\tilde{g}$ which is a product of not more than const $_{1}$ relators and of not more than const ${ }_{2}$ of commutators $\left[c_{1}, c_{2}\right]$, where $c_{1}, c_{2}$ denote some words in generators of $G_{T w}$ and in $v$ of length not exceeding const (Here $_{3}$ const $_{1}$, const 2 and const $_{3}$ are some absolute constants.) This fact immediately follows from an examination of relations of $G_{T w}$ written above The lengths of all elements $\tilde{g}_{l}$ used as some old relators of $\bar{G}_{T w}$, became bounded by an absolute constant, too

Now, as above, we use this finite presentation of $\bar{G}_{T w}$ to construct a compact $n$-dimensional manifold $M_{T w}$ such that $\pi_{1}\left(M_{T_{w}}\right)=\bar{G}_{T w}$ using the Dehn construction After this manifold is built, we are going to kill by surgeries its second homology group and to get the desired homology sphere. It is easy to see that $H_{2}\left(M_{T w}\right)$ is freely generated by 2 -cells corresponding to relators of $\bar{G}_{T w}$ of the form $[r, f]$ or $[r, v]$, where $r$ are old generators of $\bar{G}_{T w}$ and $f$ and $v$ are old and new generators of $\bar{G}_{T w}$ We are going to represent these generators of $H_{2}\left(M_{T w}\right)$ by embedded two-dimensional PL-spheres and then to kill them one by one by surgeries In order to make sure that we will not need more than $\operatorname{const}(|w|+1)$ simplices we must ensure that every of these embedded 2-spheres will contain not more than Const simplices, where Const does not depend on $|w|$. We are going to deduce this fact from the following lemma which will be proven later. (This lemma is a constructive version of the statement that the second homology group of the universal central extension of a perfect finitely presented group is trivial )

Lemma 2.2. There exist absolute constants const $_{1}$, const $\mathrm{t}_{2} \in \mathbb{N}$ with the following property Let $F$ be the free group freely generated by $f_{1}, \ldots, f_{m}$ Assume that for every $i \in\{1, \quad,, m\} m_{l} \in \mathbb{N}$ and $g_{l}, c_{l k 1}, c_{i k 2} \in F$ are such that

$$
f_{l}=g_{l} \prod_{l=1}^{m_{l}}\left[c_{i l 1}, c_{l l 2}\right]
$$

Then for every element $r \in F$ and for every $i \in\{1, \ldots, m\}$ there exists a number $q_{i} \leqq \operatorname{const}_{1} \sum_{l=1}^{m_{l}}\left(\right.$ length $\left(c_{l l 1}\right)+$ length $\left.\left(c_{l l 2}\right)\right)$ and elements $t_{i k} \in F, j \in\left\{1, \quad, q_{l}\right\}$ 
such that

and

$$
\text { length }\left(t_{i k}\right) \leqq \operatorname{const}_{2}\left(\text { length }(r)+\sum_{l=1}^{m_{i}}\left(\text { length }\left(c_{i l 1}\right)+\operatorname{length}\left(c_{i l 2}\right)\right)\right),
$$

$$
\left[r, f_{i}\right]=\left[r, g_{i}\right] \prod_{k=1}^{q_{i}}\left[\left[r, f_{i_{k}}^{\varepsilon_{k}}\right]^{\delta_{k}}, t_{i k}\right]^{\omega_{k}}\left[\left[\prod_{l=1}^{m_{i}}\left[c_{i l 1}, c_{i l 2}\right], r\right], g_{i}\right] \text {, }
$$

where $f_{i_{k}}$ are generators of $F, \varepsilon_{k}, \delta_{k}, \omega_{k}$ are equal (independently) to +1 or -1 .

Now we are going to explain how to represent the generators of $H_{2}\left(M_{T w}\right)$ represented by cells bounded by loops, corresponding to elements $\left[r_{j}, f_{i}\right]$ of the fundamental group of $M_{T w}$. (Here $f_{i}$ are old generators of this group, and $r_{j}$ are old relators.) Consider one such cell $C$. Applying Lemma 2.2 to the free group generated by old generators of $\bar{G}_{T w}$ for $\tilde{g}_{i}=g_{i}$ and $r_{j}=r$ we can assume without any loss of generality that the boundary of $C$ is the loop corresponding to the product (in some order) of commutators $\left[r_{j}, \tilde{g}_{i}\right],\left[\left[c_{i}, r_{j}\right], \tilde{g}_{i}\right]$ and not more than const $\sum_{l=1}^{m_{i}}\left(\right.$ length $\left(c_{i l 1}\right)+$ length $\left.\left(c_{i l 2}\right)\right)$ commutators of the form $\left[\left[r_{j}, f_{a}^{\varepsilon_{a}}\right]^{\delta_{a}}, t_{a}\right]^{\omega_{a}}$ (as in Lemma 2.2). (Here and below we denote for brevity $\prod_{l=1}^{m_{i}}\left[c_{i l 1}, c_{i l 2}\right]$ by $c_{i}$.) We are going to modify this product representation of $\left[r_{j}, f_{i}\right]$ in order to get rid of the commutators of the form $\left[\left[r_{j}, f_{a}^{-1}\right]^{\delta_{a}}, t_{a}\right]^{\omega_{a}}$. Recall that for every original generator $f_{a}$ of $G_{T w}$ we introduced a new generator $f_{b}$ together with the relation $f_{a} f_{b}=e$. We can replace $f_{a}^{-1}$ by $f_{b} r_{a b}^{-1}$, where $r_{a b}$ denotes the relator $f_{a} f_{b}$. We can assume without any loss of generality that $f_{a}$ is one of the original generators of $G_{T w}$ and $f_{b}$ is the generator added together with the relation $f_{a} f_{b}=e$. Since $f_{b}=f_{a}^{-1} r_{a b}$ and $f_{b}$ enters only the relation $f_{a} f_{b}=e$, we can assume that the element $\tilde{g}_{b}$ in the decomposition of $f_{b}$ is equal to $\tilde{g}_{a}^{-1} r_{a b}$ (changing, if necessary, the finite presentation of $\bar{G}_{T w}$ ). Substituting $f_{b} \tilde{g}_{b}^{-1} \tilde{g}_{a}^{-1}$ for $f_{a}^{-1}$ and using identities (b)-(f) stated in the proof of Lemma 2.2 given below we can rewrite the commutator $\left[\left[r_{j}, f_{a}^{-1}\right]^{\delta_{a}}, t_{a}\right]^{\omega_{a}}$ as a product in some order of the commutator $\left[\left[r_{j}, f_{b}\right]^{\delta_{a}}, t_{a}\right]^{\omega_{a}}$ and several commutators of the form $\left[\left[\tilde{g}_{k}^{\kappa_{k}}, r_{j}\right]^{\chi_{k}}, s_{k}\right]^{v_{k}}$, where $\tilde{g}_{k}$ are $\tilde{g}_{a}$ or $\tilde{g}_{b}, \kappa_{k}$, $\chi_{k}$ and $v_{k}$ are independently equal to +1 or to $-1, s_{k}$ are words of the length not exceeding const $\left(\right.$ length $\left(r_{j}\right)+$ length $\left.\left(t_{a}\right)+\operatorname{length}\left(\tilde{g}_{a}\right)\right)$, and const is an absolute constant. (We refer the reader interested in the details of this computation to the proof of statement B which is a part of the proof of Lemma 2.2 below. This proof involves a quite similar computation.) We can regard $C$ as an image of a continuous map $\psi$ defined on the lower hemisphere $S_{-}^{2}$ of the two-dimensional sphere $S^{2}$. We are going to extend $\psi$ to the upper hemisphere $S_{+}^{2}$ in such a manner that the resulting map $\psi: S^{2} \rightarrow M_{T w}$ will map the fundamental homology class of $S^{2}$ to the class represented by $C$. First, we map $S_{+}^{2}$ onto the wedge of twodimensional disks $B_{1}, \ldots, B_{l_{i j}}$. Their number $l_{i j}$ must be equal to the number of commutators in the product decomposition of $\left[r_{j}, f_{i}\right]$, existence of which follows from Lemma 2.2, modified as described above to get rid of the double commutators involving $f_{a}^{-1}$. Every of these disks will correspond to exactly one of the commutators $\left[r_{j}, \tilde{g}_{i}\right],\left[\left[c_{i}, r_{j}\right], \tilde{g}_{i}\right],\left[\left[\tilde{g}_{k}^{\kappa_{k}}, r_{j}\right]^{\chi_{k}}, s_{k}\right]^{v_{k}},\left[\left[r_{j}, f_{c}\right]^{\delta_{a}}, t_{a}\right]^{\omega_{a}}(c=$ a or $b)$. The part of the boundary of $S_{+}^{2}$ corresponding to one of these commutators will be mapped to the boundary of the disk, corresponding to the commutator. (Recall, that $\partial S_{+}^{2}=\partial S_{-}^{2}=\psi^{-1}(\partial C)$.) These disks then will be mapped into $M_{T w}$ as follows. The boundary of a disk $B_{k},\left(k \in\left\{1, \ldots, l_{i j}\right\}\right)$, will be mapped to a loop $M_{T w}$ representing the commutator, corresponding to $B_{k}$. (Here and below one must choose loops, representing various elements of the fundamental group of $M_{T w}$, in 
the most obvious "economical" way in the 1-skeleton of $M_{T w}$.) Assume that $B_{k}$ corresponds to the commutator $\left[\left[r_{j}, f_{a}\right]^{\delta_{a}}, t_{a}\right]^{\omega_{a}}$ for some $a$. (The case when $B_{k}$ corresponds to $\left[r_{j}, \tilde{g}_{l}\right]$, or $\left[\left[c_{l}, r_{j}\right], \tilde{g}_{l}\right]$, or $\left.\left[\left[\tilde{g}_{k}^{K_{k}}, r_{j}\right]^{\chi_{k}}, s_{k}\right]^{v_{k}}\right]$ can be treated quite similarly.) Assume that $B_{k}$ lies in the $X Y$-plane. Let us cut it by two vertical lines parallel to the $Y$-axis into three parts. Without loss of generality we can assume that $B_{k}$ is attached to the wedge of the other disks at a point of intersection of one of these vertical lines with its boundary The middle of these three parts will be first projected on a horizontal segment and then mapped onto a loop, representing $t_{a}$, in $M_{T w}$. The remaining two parts are mapped to the 2-dimensional cell in $M_{T w}$, bounded by a loop, representing $\left[r_{J}, f_{a}\right]$, with opposite orientations. (By virtue of our construction of $M_{T w}$ such a two dimensional cell exists, because $\left[r_{j}, f_{a}\right]$ is one of the relators of $\bar{G}_{T w}=\pi_{1}\left(M_{T w}\right)$.) It is clear that the so defined map $\psi$ will map the fundamental homology class of $S^{2}$ to the element of $H_{2}\left(M_{T_{w}}\right)$ represented by $C$.

An analysis of the new finite presentation of $\bar{G}_{T w}$ easily shows that $m_{i}$, length $\left(c_{i k 1}\right)$, length $\left(c_{l k 2}\right)$ and length $(r)=$ length $\left(r_{l}\right)$ do not exceed a constant independent of $|w|$ As a corollary, we see that the number $l_{l j}$ of disks in the construction of $\psi$ can be regarded as an absolute constant and that the upper bound for the length of words $t_{a}$ provided by Lemma 22 is bounded by another absolute constant.

The image of $\psi$ will not be, in general, an embedded sphere, but we can make it simlicially embedded by a small perturbation and a subdivision of the original triangulation of $M_{T w}$. A simple analysis shows that the total increase of the number of simplices due to these perturbations and subdivisions will not exceed const $_{0} \times$ the number of simplices in the image of $\psi$, where const ${ }_{0}$ is a constant depending only on the dimension of the manifold. Thus, the total increase of the number of simplices will not exceed a constant not depending on $|w|$.

Now we are going to represent by embedded spheres the generators of $H_{2}\left(M_{T w}\right)$ corresponding to relators $\left[r_{j}, v_{l}\right]$, where $r_{j}$ are old relators and $v_{i}$ are new generators (Afterwards we will kill them one by one. The generators, corresponding to relators of the form $\left[r, v_{i+1}\right]$ will be killed only after the generators, corresponding to relators $\left[r, v_{l}\right]$ ) The idea is essentially the same as before Namely, we are going to complement the map of a disk to $M_{T w}$ representing a cell bounded by a loop corresponding to $\left[r_{j}, v_{l}\right]$ by a map of another disk with the same boundary to $M_{T_{w}}$ in such a manner that the resulting map of $S^{2}$ would map the fundamental homology class of $S^{2}$ to the homology class we are going to kill. We are going to use the identities

$$
\begin{gathered}
{\left[r_{l}, v_{2}\right]=\left[r_{l}, f_{i_{1}}^{\varepsilon_{1}}\right]\left[r_{l}, f_{l_{2}}^{\varepsilon_{2}}\right]\left[\left[f_{i_{2}}^{\varepsilon_{2}}, r_{j}\right], f_{i_{1}}^{\varepsilon_{1}}\right],} \\
{\left[r_{l}, v_{l+1}\right]=\left[r_{j}, v_{l}\right]\left[r_{j}, f_{i_{l+1}}^{\varepsilon_{l+1}}\right]\left[\left[f_{i_{l+1}}^{\varepsilon_{l+1}}, r_{j}\right], v_{l}\right],}
\end{gathered}
$$

instead of Lemma 2.2. (At the moment when we will be killing the homology class corresponding to $\left[r_{j}, v_{l+1}\right]$, the homology class corresponding to $\left[r_{l}, v_{l}\right]$ is already killed and can be regarded as trivial The homology classes corresponding to $\left[r_{j}, f_{i}\right]$, will be also already killed ) Otherwise the construction is quite similar to the representation of elements of $H_{2}\left(M_{T w}\right)$ corresponding to $\left[r_{j}, f_{l}\right]$ by embedded spheres described above. After all the generators of $H_{2}\left(M_{T w}\right)$ are represented by embedded spheres, we will perform surgeries killing these generators one by one This completes the proof of Theorem 21 . 
Proof of Lemma 2.2. To prove Lemma 2.2 we perform a computation using some tricks described on p. 49 of $[\mathrm{Mn}]$. We start from the identities

$$
\begin{gathered}
\text { (a) }[v, u]=[u, v]^{-1}, \\
\text { (b) }[u, v w]=[u, v][u, w][[w, u], v],
\end{gathered}
$$

which are valid for any elements $u, v, w$ of any group. Also, we will need the following generalization of (b) which can be deduced from (b) using induction:

$$
\text { (c) }\left[u, v_{1} \ldots v_{k}\right]=\left[u, v_{1}\right] \prod_{i=2}^{k}\left[u, v_{i}\right]\left[\left[v_{i}, u\right], v_{1} \ldots v_{i-1}\right] \text {. }
$$

Now note that identity (b) implies that

$$
\left[r_{j}, f_{i}\right]=\left[r_{j}, \tilde{g}_{i} c_{i}\right]=\left[r_{j}, \tilde{g}_{i}\right]\left[r_{j}, c_{i}\right]\left[\left[c_{i}, r_{j}\right], \tilde{g}_{i}\right],
$$

where $c_{i}$ denotes $\prod_{l=1}^{m_{i}}\left[c_{i l 1}, c_{i l 2}\right]$. So, we must show that $\left[r_{j}, c_{i}\right]$ can be represented as the product of not more than const $m_{i}$ commutators of the form $\left[\left[r_{j}, f_{a}^{\varepsilon_{a}}\right]^{\delta_{a}}, t_{a}\right]^{\omega_{a}}$, where the lengths of words $t_{a}$ do not exceed the upper bound given in the text of Lemma 2.2. Using (c) we see that

$$
\begin{aligned}
{\left[r_{j}, c_{i}\right]=} & {\left[r_{j}, \prod_{l=1}^{m_{i}}\left[c_{i l 1}, c_{i l 2}\right]\right]=\left[r_{j},\left[c_{i 11}, c_{i 12}\right]\right] } \\
& \left.\times \prod_{k=2}^{m_{i}}\left(\left[r_{j},\left[c_{i k 1}, c_{i k 2}\right]\right]\left[\left[c_{i k 1}, c_{i k 2}\right], r_{j}\right], \prod_{l=1}^{k-1}\left[c_{i l 1}, c_{i l 2}\right]\right]\right) .
\end{aligned}
$$

Note that the right-hand side of this formula is the product in some order of $m_{i}$ terms of the form $\left[r_{j},\left[c_{i k 1}, c_{i k 2}\right]\right]$ for various $k$ and of $m_{i}-1$ terms of the form $\left[\left[\tilde{w}, r_{j}\right], \bar{w}\right]$, where $\tilde{w}$ are words of length not exceeding $2\left(\right.$ length $\left(c_{i k 1}\right)+$ length $\left.\left(c_{i k 2}\right)\right)$ and $\bar{w}$ are words of length not exceeding $2 \sum_{k=1}^{m_{l}}\left(\operatorname{length}\left(c_{i k 1}\right)+\operatorname{length}\left(c_{i k 2}\right)\right)$. Now we see that Lemma 2.2 would follow from the validity of the following two statements:

A. There exist absolute constants $N_{0}$ and const such that for any $y_{1}, y_{2} \in F$ the commutator $\left[r_{j},\left[y_{1}, y_{2}\right]\right]$ can be represented as the product $\prod_{k=1}^{N_{0}}\left[\left[r_{j}, z_{k 1}\right]^{\delta_{k}}, z_{k 2}\right]^{\omega_{k}}$, where $\delta_{k}$ and $\omega_{k}$ are (independently) equal to +1 or to -1 and $z_{k 1}, z_{k 2}$ are words in generators of $F$ and their inverses such that length $\left(z_{k 1}\right) \leqq \operatorname{const}\left(\right.$ length $\left(y_{1}\right)+$ length $\left.\left(y_{2}\right)\right)$, length $\left(z_{k 2}\right) \leqq \operatorname{const}\left(\right.$ length $\left(y_{1}\right)+$ length $\left(y_{2}\right)+$ length $\left.\left(r_{j}\right)\right)$.

B. There exists an absolute constant const such that for any $z_{1}, z_{2} \in F$ the commutators $\left[\left[r_{j}, z_{1}\right], z_{2}\right]$ and $\left[\left[z_{1}, r_{j}\right], z_{2}\right]$ can be represented as products of $k \leqq$ const length $\left(z_{1}\right)$ commutators of the form $\left[\left[r_{j}, f_{a}^{\varepsilon_{a}}\right]^{\delta_{a}}, t_{a}\right]^{\omega_{a}}, a \in\{1, \ldots, k\}$, where $t_{a}$ are arbitrary words of length not exceeding const $\left(\right.$ length $\left(r_{j}\right)+\operatorname{length}\left(z_{1}\right)+$ length $\left(z_{2}\right)$ ), and $\omega_{a}, \varepsilon_{a}$ and $\delta_{a}$ are equal to +1 or to -1 .

First, we are going to prove the statement B. We will prove the existence of the product decomposition only for the commutator $\left[\left[r_{j}, z_{1}\right], z_{2}\right]$. The proof for the commutator $\left[\left[z_{1}, r_{j}\right], z_{2}\right]$ is similar. Let $z_{1}=\prod_{k=1}^{\text {length }\left(z_{1}\right)} f_{i_{k}}^{\varepsilon_{i_{k}}}$, where $f_{i_{k}}$ are generators of $F$ and $\varepsilon_{i_{k}}=1$ or -1 . Note that the identity (c) implies that

$$
\left[\left[r_{j}, z_{1}\right], z_{2}\right]=\left[\left[r_{j}, f_{i_{1}}^{\varepsilon_{i_{1}}}\right] \prod_{k=2}^{\text {length }\left(z_{1}\right)}\left(\left[r_{j}, f_{i_{k}}^{\varepsilon_{i_{k}}}\right]\left[\left[f_{i_{k}}^{\varepsilon_{i_{k}}}, r_{j}\right], \prod_{l=1}^{k-1} f_{i_{l}}^{\varepsilon_{l_{l}}}\right]\right), z_{2}\right] .
$$


Denote the commutators $\left[r_{j}, f_{i_{1}}^{\varepsilon_{l_{1}}}\right],\left[r_{j}, f_{i_{2}}^{\varepsilon_{i_{2}}}\right],\left[\left[f_{i_{2}}^{\varepsilon_{i_{2}}}, r_{j}\right], f_{l_{1}}^{\varepsilon_{i_{1}}}\right], \ldots$ in the right-hand side of this equality by $w_{1}, w_{2}, w_{3}, \ldots$ In these notations

$$
\left[\left[r_{j}, z_{1}\right], z_{2}\right]=\left[\prod_{k=1}^{2 \text { length }\left(z_{1}\right)-1} w_{k}, z_{2}\right]
$$

Applying identities (a), (c) and once again (a) we see that

$$
\begin{aligned}
{\left[\left[r_{j}, z_{1}\right], z_{2}\right]=} & \prod_{k=1}^{2 \text { length }\left(z_{1}\right)-2}\left(\left[\prod_{l=1}^{2 \text { length }\left(z_{1}\right)-k-1} w_{l},\left[w_{2 l e n g t h}\left(z_{1}\right)-k, z_{2}\right]\right]\right. \\
& \left.\times\left[w_{2 l e n g t h}\left(z_{1}\right)-k, z_{2}\right]\right)\left[w_{1}, z_{2}\right] .
\end{aligned}
$$

Denote $\prod_{l=1}^{k-1} w_{l}$ by $\hat{w}_{k}$. The identity (c) implies that

$$
\tilde{w}_{2 l}=\left[r_{j}, \prod_{s=1}^{l-1} f_{i_{s}}^{\varepsilon_{i_{s}}}\right], \quad \tilde{w}_{2 l+1}=\left[r_{j}, \prod_{s=1}^{l-1} f_{i_{s}}^{\varepsilon_{i_{s}}}\right]\left[r_{j}, f_{i_{l}}^{\varepsilon_{i_{l}}}\right] .
$$

Hence the elements of $F$ represented by the words $\tilde{w}_{k}$ can be represented by words of length bounded from above by $4\left(\right.$ length $\left(r_{j}\right)+$ length $\left.\left(z_{1}\right)\right)$

Thus, we represented $\left[\left[r_{j}, z_{1}\right], z_{2}\right]$ as the product of 4 length $\left(z_{1}\right)-3$ commutators of the following four types $\left[\left[r_{j}, f_{i_{k}}^{\varepsilon_{i_{k}}}\right], z_{2}\right],\left[\left[\left[f_{i_{k}}^{\varepsilon_{l_{k}}}, r_{j}\right], \prod_{l=1}^{k-1} f_{i_{l}}^{\varepsilon_{l_{l}}}\right], z_{2}\right],\left[\tilde{w}_{k},\left[\left[r_{j}, f_{i_{k}}^{\varepsilon_{i_{l}}}\right]\right.\right.$, $\left.\left.z_{2}\right]\right]$ and $\left[\tilde{w}_{k},\left[\left[\left[f_{i_{k}}^{\varepsilon_{i_{k}}}, r_{j}\right], \prod_{l=1}^{k-1} f_{i_{l}}^{\varepsilon_{i_{l}}}\right], z_{2}\right]\right]$ In order to replace triple and quadruple commutators by double commutators one can use the following identities:

$$
\begin{gathered}
\text { (d) }[[[r, x], y], z]=[[r, x], y][[r, x], z y]^{-1}[[r, x], z], \\
(e)[[y,[r, x]], z]=[[r, x], y]^{-1}[[r, x], z]^{-1}[[r, x], z y], \\
(f) \quad[u,[[[x, r], y], z]]=[u,[[x, r], y]][[[x, r], y], u z][z,[[x, r], y]],
\end{gathered}
$$

valid for any elements $r, x, y, z, u$ of any group (These identities follow from the identity (b) above ) These identities can be applied in the obvious way to replace the triple and quadruple commutators entering the obtained product representation of $\left.\left[r_{j}, z_{1}\right], z_{2}\right]$ by the double commutators This completes the proof of the statement $\mathrm{B}$.

To prove the statement A formulated above note that it is sufficient to prove the existence of the product decomposition with the desired properties for $\left[r_{j},\left[y_{1}, y_{2}\right]\right]$ $\left[y_{1},\left[y_{2}, r_{j}\right]\right]\left[y_{2},\left[r_{j}, y_{1}\right]\right]$ instead of $\left[r_{j},\left[y_{1}, y_{2}\right]\right]$ Applying identities (a) and (b) one can easily see that

$$
\begin{gathered}
{\left[r_{j},\left[y_{1}, y_{2}\right]\right]\left[y_{1},\left[y_{2}, r_{j}\right]\right]\left[y_{2},\left[r_{j}, y_{1}\right]\right]=\left[r_{j} y_{1}, y_{2}\right]\left[y_{2}, r_{j}\right]\left[y_{2}, y_{1}\right]} \\
\times\left[y_{1} y_{2}, r_{j}\right]\left[r_{j}, y_{1}\right]\left[r_{l}, y_{2}\right]\left[y_{2} r_{l}, y_{1}\right]\left[y_{1}, y_{2}\right]\left[y_{1}, r_{j}\right]
\end{gathered}
$$

For brevity let us call the words of the form $\left[\left[r_{j}, z_{1}\right]^{\delta}, z_{2}\right]^{\varepsilon},(\delta, \varepsilon=1$ or -1$)$ allowed. We are going to show that the right-hand side of $(*)$ can be represented as a product of several allowed words To achieve this goal we can transform the right-hand side of $(*)$ permuting terms of the form $\left[r_{j}, y\right]$ or $\left[y, r_{j}\right]$, where $y$ can be an arbitrary word (in particular, $y_{1}$ or $y_{2}$ ), with any word $y_{0}$ and taking into account that the allowed words of the form $\left[\left[r_{j}, y\right], y_{0}\right]$ or $\left[\left[y, r_{j}\right], y_{0}\right]$ or their inverses also appear in the expression as the result of the transformation. These allowed commutators 
then also can be permuted with any term in the resulting product because of the identities (d) and (e) above. (Of course, new commutators of the allowed type arise as the result of such permutations.) These permutations of commutators in the righthand side of $(*)$ are aimed to make the commutators $\left[r_{j}, y_{1}\right]$ and $\left[y_{1}, r_{j}\right],\left[r_{j}, y_{2}\right]$ and $\left[y_{2}, r_{j}\right]$ in the right-hand side of $(*)$ to cancel each other. It is not difficult to exhibit explicitly a sequence of several such permutations (and reductions) transforming the right-hand side of $(*)$ into the product of $\left[r_{j} y_{1}, y_{2}\right]\left[y_{2}, y_{1}\right]\left[y_{2} r_{j}, y_{1}\right]\left[y_{1}, y_{2}\right]\left[y_{1} y_{2}, r_{j}\right]$ and several allowed words appearing as the result of these transformations. These allowed words in principle can be written explicitly and their length does not exceed const $\left(\right.$ length $\left(y_{1}\right)+$ length $\left(y_{2}\right)+$ length $\left.\left(r_{j}\right)\right)$ for a certain absolute constant const.

Observe that

$$
\left[r_{j} y_{1}, y_{2}\right]\left[y_{2} r_{j}, y_{1}\right]\left[y_{1} y_{2}, r_{j}\right]=e \text {. }
$$

Thus, to complete the proof of statement B it is sufficient to exhibit a sequence of several permutations of commutators of the form $\left[r_{j}, y\right]$ or $\left[y, r_{j}\right]$ with arbitrary terms of the product transforming the product $\left[y_{2}, y_{1}\right]\left[y_{2} r_{j}, y_{1}\right]\left[y_{1}, y_{2}\right]$ into $\left[y_{2} r_{j}, y_{1}\right]$. (Of course, several allowed commutators will appear as the result of such permutations. But, as it was noted above, these new allowed commutators can be "moved aside" of the triple product using appropriate permutations and the identities (d) and (e).)

Note that

$$
\left[y_{2}, y_{1}\right]\left[y_{2} r_{j}, y_{1}\right]\left[y_{1}, y_{2}\right]=\left[y_{2}, y_{1}\right] y_{2}\left[r_{j}, y_{1}\right] y_{2}^{-1} \text {, }
$$

and

$$
\left[y_{2} r_{j}, y_{1}\right]=y_{2}\left[r_{j}, y_{1}\right] y_{2}^{-1}\left[y_{2}, y_{1}\right] \text {. }
$$

Permuting $\left[r_{j}, y_{1}\right]$ with $y_{2}^{-1}$ in the first equality and also permuting $\left[r_{j}, y_{1}\right]$ first with $y_{2}$ and then with $\left[y_{2}, y_{1}\right]$ in the second equality, we obtain the desired result. This completes the proof of the statement $\mathrm{A}$ and, thus, the lemma.

\section{Algorithmic Information Theory and Proof of Theorem 1.1}

Before proving Theorem 1.1 we would like to recall some facts about the Kolmogorov complexity. The Kolmogorov complexity was introduced independently by Solomonoff, Kolmogorov and Chaitin. We refer the reader to reviews [ZL, LV], books [C and $\mathrm{M}$ ], and paper [D] for discussions of the Kolmogorov complexity. Here we will use only the notion of Kolmogorov complexity for decision problems. Informally, this notion can be explained as follows:

Assume that we deal with a decision problem. This can be the problem to find out whether or not a given Turing machine starting to work with the empty tape will eventually halt, or the problem whether or not a given abstract simplicial complex is a triangulation of some given fixed compact $P L$-manifold. This problem can be unsolvable. Further, there exists a natural complexity parameter such that when its value is $\leqq N$ there is only a finite set of the instances of the problem. In the first example we could regard the number of states of Turing machines as such a parameter. In the second case the number of simplices can be considered as the complexity parameter. The unsolvability of these problems implies that there is no algorithm which for a given $N$ and a given problem instance of complexity $\leqq N$ solves the problem. However, if one is permitted to ask for additional information (the amount of which can depend on $N$ ), then the problem clearly can be solved. 
(The additional information will be regarded as the input data for the algorithm solving the decision problem). The most obvious way is just to use the list of all answers as the additional information. Usually, however, one is able to solve the decision problem using much less information. If the decision problem is solvable one does not need any additional information at all. Let us regard the minimal amount of bits of information necessary to solve the decision problem $D$ for all instances of complexity $\leqq N$ as a function $K(D, N)$ of $N$ Furthermore, one can modify this definition imposing the following restriction on the considered programs: Let $\lambda$ be a fixed recursive function. One regards as permitted, only programs which perform $\leqq \lambda(N)$ elementary operations to solve the decision problem for any given problem instance of complexity $\leqq N$ This restriction on the considered programs can lead (and sometimes leads) to a considerable increase of the minimal amount of input data required to solve $D$. Denote the minimal amount of input data required to solve $D$ for any instance of length $\leqq N$ performing not more than $\lambda(N)$ elementary operations by $K^{(\hat{\lambda})}(D, N)$.

The model of computation used here, in principle, can be defined as follows. Take any programming language of high level (say, FORTRAN or PASCAL) and strip it of all data types except for the integer type. This will be the language in which it is permitted to write the discussed programs. The notion of "minimality" of the input data requires the following clarification. it is defined only up to a constant. That is, it is possible to show that there exists a program $\pi_{0}$ requiring $K_{\pi_{0}}(D, N)$ bits of additional input information able to solve $D$ for any instance of $D$ of complexity $\leqq N$ such that for any other program $\pi_{1}$ the corresponding amount of bits $K_{\pi_{1}}(D, N)$ satisfies the inequality

$$
K_{\pi_{0}}(D, N) \leqq K_{\pi_{1}}(D, N)+C\left(\pi_{0}, \pi_{1}\right),
$$

where $C\left(\pi_{0}, \pi_{1}\right)$ is a constant which does not depend on $N$ (but depends on $\pi_{0}, \pi_{1}$ and the used model of computations). Thus, it is natural to regard $K(D, N)$ as the equivalence class of functions with respect to the equivalence relation " $\approx$ " defined as follows: $f \approx g$ iff $\exists C$ such that for any $N|f(N)-g(N)| \leqq C$. Then $K(D, N)$ will be a rigorously defined equivalence class Moreover, it will not depend on the choice of a model of computation in a wide class of models (including the one considered above and also more traditional models considered in the references above) The reason is that one can write in one language a finite program interpreting the commands of another equivalent language So no new input information will be necessary. Similarly, for any model of computation $\rho$ and any recursive function $\lambda$ we can define the equivalence class $K_{\rho}^{(i)}(D, N)$ as the equivalence class of the functions of $N$ defined as the minimal number of bits of additional input information necessary to be able to solve $D$ for all instances of complexity $\leqq N$ using a program performing not more than $\lambda(N)$ operations.

Now let us be more formal. Following [ZL, M, B] define for any partial recursive function $\phi: \mathbb{N}^{2} \rightarrow \mathbb{N}$ and a set $M$ of natural numbers the Kolmogorov complexity $K_{\phi}(M, N)$ as the minimal number of binary digits of a number $p$ such that for any natural $x \leqq N$,

$$
\phi(p, x)=\left\{\begin{array}{ll}
1, & \text { if } x \in M \\
0, & \text { if } x \notin M
\end{array}\right. \text {. }
$$

If no such $p$ exists we set $K_{\phi}(M, N)=+\infty$. It is well-known (cf [M, Theorem 9.2, $\mathrm{p}$ 226], [ZL]) that there exists a partial recursive function $R(p, x)$ such that for any 
other partial recursive function $\psi(p, x)$ and any $M$,

$$
K_{R}(M, N) \leqq K_{\psi}(M, N)+C(R, \psi),
$$

where $C(R, \psi)$ is a constant independent of $M$ and $N$. Intuitively, $R$ can be viewed as a system of programming, and $p$ as a number, the binary expansion of which codes both the program and the additional oracle information required to solve for $x$ the decision problem $D$ : "Is $x$ in $M$ ?" Following [M, p. 226], let us describe a construction of one such $R$. Let $v: \mathbb{N} \times \mathbb{N} \rightarrow \mathbb{N}$ be a recursive imbedding function which has a recursive inverse function and which satisfies the following linear growth condition in one of its arguments: $v(k, j) \leqq k \beta(j)$, for all $k, j$ and some function $\beta$. For example, we can take $v(k, j)=(2 k-1) 2^{j-1}$. Let $\mu: \mathbb{N} \rightarrow \mathbb{N} \times \mathbb{N}$ be the inverse function of $v$. Consider a Gödel numbering by consecutive integers of all Turing machines working with two input binary sequences. (To be precise one can assume that at the beginning of computations one of these sequences is to the left of the scanning head and another one is to the right of the scanning head.) The function $R(p, x)$ can be described as follows: Consider $\mu(p)=\left(\mu_{1}(p), \mu_{2}(p)\right)$. $R(p, x)$ is the result of the computation performed by the Turing machine coded by $\mu_{2}(p)$ starting from the inputs $\mu_{1}(p), x$. (Informally, $\mu_{1}(p)$ can be interpreted as the oracle information and $x$ as the data for the considered algorithmic problem.) If this computation does not stop, then $R(p, x)$ is not defined. Define $t(p, x)$ as the time required to the Turing machine coded by $\mu_{2}(p)$ and starting to work with inputs $\mu_{1}(p), x$ to complete the computation. If this computation does not stop then we assume that $t(p, x)=\infty$. Let $\lambda$, compl be arbitrary recursive functions. Assume that compl is increasing. Define $K_{R}^{(\lambda)}(M, N)$ as the minimal length of the numbers $p$ (written in the binary system) such that for any natural number $x$ such that $\operatorname{compl}(x) \leqq N$

(a) $R(p, x)=\left\{\begin{array}{ll}1, & \text { if } x \in M \\ 0, & \text { if } x \notin M\end{array}\right.$,

(b) $t(p, x) \leqq \lambda(N)$.

We will call $K_{R}^{(\lambda)}(M, N)$ the time-bounded Kolmogorov complexity or $\lambda$-bounded Kolmogorov complexity. Of course $K_{R}^{(\lambda)}(M, N)$ depends also on the choice of compl. In the standard definitions of time-bounded Kolmogorov complexity $\operatorname{compl}(x)=x$. Here we will use mostly $\operatorname{compl}(x)=\operatorname{length}(x)=\left[\log _{2}(x)\right]+1$. Also, when we will consider the recognition of a PL-manifold problem and $x$ will code a triangulation, $\operatorname{compl}(x)$ will be the number of simplices in this triangulation. (The relationship between $\lambda$-bounded Kolmogorov complexities for different choices of compl is entirely obvious, so the choice of a way to measure complexity of the input is just a matter of convenience when we discuss decision problems involving complicated objects coded by numbers.) It is clear that the definition of time-bounded Kolmogorov complexity is a formalization of the informal definition given at the beginning of this section and in the introduction. Similarly, one can define very close notions of Kolmogorov complexity and time-bounded Kolmogorov complexity of finite and infinite binary sequences. Let $m=\left(m_{1}, \ldots\right)$ be a sequence of 0 's and 1's of the length at least $N$. Define the Kolmogorov complexity $K_{R}(m, N)$ (resp. $\lambda$-bounded Kolmogorov complexity $K_{R}^{(\lambda)}(m, N)$ ) as the number of digits in the binary representation of the minimal number $p$ such that for all $x \leqq N R(p, x)=m_{x}$ (resp. $R(p, x)=m_{x}$ and in addition $\left.t(p, x) \leqq \lambda(N)\right)$. Note that the $\lambda$-bounded Kolmogorov complexity of the decision problem "Is $x$ in $M$ ?" for $\operatorname{compl}(x)=x$ is equal to the 
$\lambda$-bounded Kolmogorov complexity of the sequence of values of the characteristic function of $M(\subset \mathbb{N})$.

Barzdin ([B, Theorem 3]) discovered the existence of a recursively enumerable set $H \subset \mathbb{N}$ such that for any recursive function $\lambda$ for all sufficiently large $N$ the $\lambda$-bounded Kolmogorov complexity of the recognition problem whether or not $k$ is an element of $H$ for all $k \leqq N$ (i.e for $\operatorname{compl}(x)=x$ ) is bounded from below by $\frac{N}{c(\lambda)}$ for some constant $c(\lambda)$ (depending on $\lambda$ and $R$ but not on $N$ ). If we will consider the length of the binary expansion of $k$ instead of the number $k$ itself as the complexity (that is, if we take $\operatorname{compl}(x)=\left[\log _{2}(x)\right]+1$ ), then for all sufficiently large $N$,

$$
K_{R}^{(\hat{\lambda})}(H, N) \geqq 2^{N} / c(\hat{\lambda}) .
$$

The left-hand side of (2) is the time-bounded Kolmogorov complexity with time resources bounded by $\lambda$ of the problem of recognition whether or not $k \in H$ for integer numbers $k$ having the binary expansion of length $\leqq N$ A construction of the characteristic function of such a set $H$ can be found in the proof of Theorem 2.5 in [ZL]. (A very close result with a similar proof can be found in [D] (Theorem 9).) More precisely, in $[\mathrm{ZL}]$ the authors construct an infinite binary sequence such that. 1) For any recursive $\lambda$ for all sufficiently large $N$ the $\lambda$-bounded Kolmogorov complexity of this sequence is not less than $N / c(\lambda)$; and 2) This binary sequence is the sequence of values of the characteristic function of a recursively enumerable set.

For the sake of completeness we are going to outline a construction of such an infinite binary sequence (Our exposition follows [ZL, p 97] and [D].) Only the following two obvious properties of the time-bounded Kolmogorov complexity are used First, the time-bounded Kolmogorov complexity majorizes the "standard" Kolmogorov complexity (without restrictions on time) Secondly, there exists an algorithm computing $K_{R}^{(i)}(m, N)$ for any given $N$, any given finite binary sequence $m$ of length $\geqq N$ and using a given value of $\lambda(N)$. (Indeed, we can find the minimal $p$ trying one by one all $p$ 's For each $p$ we find the Turing machine coded by $\mu_{2}(p)$ For every $x$ such that $x \leqq N$ we apply this machine to $\mu_{1}(p), x$ and wait for the time $\lambda(N)$ If for some $x$ the computation does not stop in this time, then we pass to the next $p$. Otherwise, we compare the results of computations with numbers $m_{x}$. In the case of coincidence we can be sure that we found the minimal $p$ ) As a corollary of these two properties, there exists an algorithm which for every $N$ and a partially recursive function $\lambda$ defined on $N$ finds a binary sequence of length $\leqq N$ and of the $\lambda$-bounded Kolmogorov complexity not less than $N$. (The existence of such a sequence follows from the fact that there are not more than $2^{N-1}$ binary sequences $m$ of length $N$ for which there exists $p<2^{N-1}$ such that $R(p, x)=m_{x}$ for all $x=1, \ldots, N$.) The required infinite binary sequence can be now constructed by a diagonalization construction using these finite sequences of high time-bounded Kolmogorov complexity. Namely, the sequence will consist of pieces written one after another The length of the $i^{\text {th }}$ piece is equal to $2^{i}$. This piece is filled by 0's and 1's as follows Denote the maximal number $k$ such that $2^{k}$ divides $i$ by $k(i)$ (Observe that for every $k$ the set of $j$ such that $k(j)=k$ forms an arithmetic progression.) For every $j$ define a partial recursive function $\lambda_{j}$ by the formula $\lambda_{j}(x)=R(k(j)+1, x)$ If $\lambda_{l}\left(2^{l}\right)$ is not defined, then we fill the $i^{\text {th }}$ piece of the sequence by zeroes. Otherwise, we place in the $i^{\text {th }}$ piece the binary sequence of length $2^{l}$ and of $\lambda_{l}$-bounded Kolmogorov complexity $\geqq 2^{l}$ constructed by the mentioned above algorithm Obviously, the resulting infinite sequence is a sequence of values of the characteristic function of a recursively enumerable set $H$ Observe, 
that every partial recursive function $\lambda$ will be among functions $\lambda_{j}$. Moreover, the set of $j$ such that $\lambda=\lambda_{j}$ contains an arithmetic progression. It is not difficult to prove using this observation that the constructed recursively enumerable set $H$ satisfies the inequality (2) for every recursive function $\lambda$.

Consider a Turing machine $\tau_{0}$ such that the set of binary expansions of elements from the set $H$ is precisely the set of inputs for which $\tau_{0}$ halts. Consider the halting problem for $\tau_{0}$ (i.e. the problem whether or not for an input binary sequence $w \tau_{0}$ starting to work with $w$ will eventually halt.) Obviously, the left-hand side of (2) can be interpreted as the $\lambda$-bounded Kolmogorov complexity of the halting problem for $\tau_{0}$ for input (binary) words of length $\leqq N$. Thus, the halting problem for $\tau_{0}$ has $\lambda$-bounded Kolmogorov complexity bounded from below by $2^{N} / c(\lambda)$ for all sufficiently large $N$.

Now we can outline the idea of the proof of Theorem 1.1. Let an effective enumeration of finite simplicial complexes increasing with the number of simplices be fixed. Let $H_{M^{n}}$ denote the set of numerals of simplicial complexes such that their spaces are $P L$-homeomorphic to $M^{n}$. Consider the number of simplices $N$ in a simplicial complex as the complexity parameter in the algorithmic problem of recognizing whether or not the space of a given simplicial complex is PL-homeomorphic to $M^{n}$. If $n \geqq 5$ then the quantitative version of the algorithmic unrecognizability of $S^{n}$ (Theorem 2.1) immediately implies the exponential lower bound for $K_{R}^{(\lambda)}\left(H_{M^{n}}, N\right)$ (Lemma 3.1(a)). Also, in the case, when $n \geqq 4$ and $M^{n}$ is a connected sum of a compact PL-manifold $N^{n}$ and a sufficiently large number of copies of $S^{2} \times S^{n-2}$ one can derive the same exponential lower bound for $K_{R}^{(\lambda)}\left(H_{\left.M^{n}, N\right)}\right.$ reviewing the Markov proof of unrecognizability of $M^{n}$ (Lemma 3.1(b)). (Of course, only for $n=4$ this bound does not immediately follow from Theorem 2.1.) On the other hand we will find for an appropriate recursive function $\lambda$ an upper bound for $K_{R}^{(\lambda)}\left(H_{M^{n}}, N\right)$ linearly growing with $m_{A, M}(N)$ (Lemma 3.2). The juxtaposition of these lower and upper bounds implies Theorem 1.1.

Lemma 3.1. (a) For any $n \geqq 5$ there exists a constant $C_{0}(n)>1$ such that for any compact $n$-dimensional $P L$ manifold $M$ and for any recursive function $\lambda$ there exists $N_{0}$ (depending on $M, \lambda$ and $R$ ) such that for any $N \geqq N_{0}$,

$$
K_{R}^{(\lambda)}\left(H_{M}, N\right)>C_{0}^{N}(n) .
$$

(b) There exists $k$ such that for any $n \geqq 4$ there exists a real constant $C_{0}(n)>1$ with the following property: For any manifold $M$ of dimension $n \geqq 4$ representable as the connected sum $N^{n} \# k S^{2} \times S^{n-2}$, where $N^{n}$ is a compact $n$-dimensional PL-manifold, there exists $N_{0}$ (depending on $\lambda, R$ and $N^{n}$ ) such that for all $N \geqq N_{0}$,

$$
K_{R}^{(\lambda)}\left(H_{M}, N\right)>C_{0}^{N}(n) .
$$

Proof. (a) Theorem 2.1 implies that the halting problem for any fixed Turing machine and, in particular, for the defined above Turing machine $\tau_{0}$ can be reduced to a problem of recognizing whether or not a given triangulation is a triangulation of $M$. Moreover, when the Turing machine is fixed, the number of simplices in this triangulation is bounded from above by a linear function of the length of the input $w$ in the halting problem for the Turing machine. Denote by Halt $\tau_{\tau_{0}}$ the set of inputs of $\tau_{0}$ for which it eventually halts, by $\bar{\lambda}_{\tau_{0}}(N)$ the maximal time of work of the algorithm defined in Theorem 2.1 on a set of input data, consisting of the manifold 
$M$, the Turing machine $\tau_{0}$ and any of its inputs $w$ of the length not exceeding $N$. Then for any recursive function $\lambda$ such that $\lambda>\bar{\lambda}_{\tau_{0}}$ inequality (2) implies that for all sufficiently large $N$,

$$
2^{N} / c(\lambda) \leqq K_{R}^{(i)}\left(\operatorname{Halt}_{\tau_{0}}, N\right) \leqq K_{R}^{\left(i-\bar{\lambda}_{0}\right)}\left(H_{M}, c_{n}\left(\tau_{0}\right) N+c_{n}\left(\tau_{0}\right)+|M|\right)+\text { const } .
$$

(Here const is a constant not depending on $N$ and $\lambda, c_{n}\left(\tau_{0}\right)$ is the value of the constant $c_{n}(T)$ defined in the text of Theorem 2.1 for $T=\tau_{0}$. Of course, $c_{n}\left(\tau_{0}\right)$ does not depend on $\lambda$.) This double inequality implies that

$$
K_{R}^{(\hat{\lambda})}\left(H_{M}, N\right) \geqq 2^{\left(N-c_{n}\left(\tau_{0}\right)-|M|\right) / c_{n}\left(\tau_{0}\right)} / c\left(\lambda+\bar{\lambda}_{\tau_{0}}\right)-\text { const },
$$

which proves part (a) of the lemma.

(b) For any $n \geqq 5$ this part of the lemma is an immediate corollary of part (a) However, since we still need to prove part (b) for $n=4$ we will give a proof not referring to Theorem 2.1 or any other material in Sect. 2 and valid for any $n \geqq 4$. Our proof will be based on a quantitative analysis of the Markov proof explained in [BHP] of the algorithmic unsolvability of the $P L$-homeomorphism problem for compact $P L$-manifolds of dimension $n \geqq 4$. Since this proof uses the unsolvability of the triviality problem for finitely presented groups (the Adyan-Rabin theorem) we will briefly review a proof of this result too.

Step 1 Let a Turing machine $T$ and its input word $w$ be given. There are several ways to effectively construct a finite presentation of a group $G(T)$, which depends only on $T$ but not on $w$, and its element $g(w)$, such that $g(w)$ is trivial in $G(T)$ if and only if $T$ starting to work with the input $w$ will eventually halt For example, one such construction is described in [R]. Note that the number of generators, the number of relations, and the length of relators in the finite presentation of the group $G(T)$ constructed according to [R] are bounded by a constant depending on $T$, but of course not on $w$, and the length of $g(w)$ is bounded by const $_{2} N$, where $N$ is the length of $w$ and const $_{2}$ is a factor independent of $w$. On this step we apply this construction to the defined above Turing machine $\tau_{0}$.

Step 2 We intend to construct starting from a given finite presentation of a group $G$ and an element $g \in G$ a finite presentation of a group $G_{g}$ in such a manner that $g=e$ in $G$ if and only if $G_{g}$ is trivial To achieve this goal we use the effective procedure described in [Mi], pp 13-15 When we apply this construction to the finite presentation $G\left(\tau_{0}\right)$ and the word $g(w)$ constructed on the previous stage, we obtain a finite presentation of a group $G\left(\tau_{0}\right)_{g(w)}$. The number Gen of generators and the number Rel of relations in this finite presentation do not depend on $g(w)$ (see [Mi]) and, hence, on $N$ The length of every relator in this finite presentation is bounded by $\operatorname{const}_{3} N$, where $N$ is the length of $w$ and const $_{3}$ is a constant factor

Step 3 Now we add to the finite presentation of $G\left(\tau_{0}\right)_{g(w)}$ several empty relations According to the exposition of the Markov construction in [BHP], it is necessary to add 4 Rel $+5 G e n$ empty relations, where Rel and Gen are, correspondingly, the numbers of relations and generators of the finite presentation (Actually, one can add just Gen empty relations, as in [F] But this improvement is of no importance for us.) Afterwards we build a hypersurface in $\mathbb{R}^{n+1}$ ( $n$ is any number $\geqq 4$ ) diffeomorphic to the connected sum of Gen copies of $S^{1} \times S^{n-1}$ Then one realizes 
relators of $G\left(\tau_{0}\right)_{g(w)}$ by pairwise non-intersecting embedded smooth closed curves in this hypersurface and performs surgeries killing the elements of the fundamental group of the hypersurface corresponding to the relations. The fundamental group of the resulting manifold is $G\left(\tau_{0}\right)_{g(w)}$. It is clear that this manifold can be triangulated using not more than const ${ }_{4} N$ simplices. Let $k$ be equal to $4 \mathrm{Gen}+5 \mathrm{Rel}$. (Our choice of $k$ is not optimal. This value can be improved by adding only Gen empty relations instead of $4 \mathrm{Rel}+5 \mathrm{Gen}$ to the finite presentation of $G\left(\tau_{0}\right)_{g(w)}$, as in [F]. This version of Markov's construction enables one to take $k=R e l$.) After $\tau_{0}$ and the specific construction of $G\left(\tau_{0}\right)_{g(w)}$ were fixed, $k$ is just a constant. It is shown in [BHP] that the resulting manifold $M_{w}^{n}$ will be $P L$-homeomorphic to the connected sum of $k$ copies of $S^{2} \times S^{n-2}$ if and only if $\pi_{1}\left(M_{w}^{n}\right)$ is trivial, or, equivalently, if $\tau_{0}$ eventually halts when applied to the input $w$. Thus, the halting problem for $\tau_{0}$ for input binary sequences of length $\leqq N$ can be effectively reduced to the problem of recognition of the connected sum of any compact $P L$-manifold $N^{n}$ and $k$ copies of $S^{2} \times S^{n-2}(n \geqq 4)$ among triangulated $P L$-manifolds with $\leqq$ const $_{4} N+\left|N^{n}\right|$ simplices. Here const ${ }_{4}$ depends only on $n$ and $\left|N^{n}\right|$ denotes the number of simplices in an arbitrary fixed triangulation of the manifold $N^{n}$. Now the lemma follows immediately from the Barzdin lower estimate (2) for the time-bounded Kolmogorov complexity of the halting problem for $\tau_{0}$.

The next lemma provides an upper estimate of $K_{R}^{\left(\lambda_{A}\right)}\left(H_{M}, N\right)$ for an appropriate recursive function $\lambda_{A}(N)$ :

Lemma 3.2. There exist a recursive function $\lambda_{A}(N)$ and a constant $C$ independent of $N$ such that

$$
K_{R}^{\left(\lambda_{A}\right)}\left(H_{M}, N\right) \leqq C N \ln N \cdot m_{A, M}(N) .
$$

Proof We must give an upper estimate for the number of bits of oracle information sufficient to solve the problem of recognition of $M$ among simplicial complexes with $\leqq N$ simplices in a time bounded by a recursive function of $N$. This information will be the following: I require a list $L_{0}$ of triangulations representing all $m_{A, M}(N)$ minimal elements of $T_{A, M}(N)$ and containing precisely one triangulation from every minimal element. (Recall, that these minimal elements are sets of triangulations of $M$ with $\leqq N$ simplices.) Observe, that a triangulation $T$ of $M$ with $\leqq N$ simplices can be coded as follows: First, we list the number of vertices in $T$. Assume that all vertices of $T$ are numbered by consecutive natural numbers starting from 1 . We list for any face of $T$ of the maximal dimension numbers of all vertices forming this face. Thus, we obtain not more than $N$ lists of $(n+1)$ numbers from 1 to not more than $N(n$ is the dimension of $M)$. Thus, in total we have $1+(n+1) N$ numbers and the binary expansion of each number contains not more than $\log _{2} N+1$ digits. Of course, not every such set of data corresponds to a triangulation of $M$ and there are many possible ways to assign such set of data to any element of $m_{A, M}(N)$. But if such a set of data defines an element of $m_{A, M}(N)$, it defines this element uniquely.

These data describing the list $L_{0}$ and containing not more than $C N \ln N m_{A, M}(N)$ bits of information (where $C$ does not depend on $N$ ) are sufficient to solve the problem whether or not a given simplicial complex with $\leqq N$ simplices is a triangulation of $M$ in a time, bounded from above by a recursive function of $N$. Indeed, first construct the complete list of all triangulations of $M$ with less than or equal to $N$ simplices using the following algorithm: Apply the algorithm $A$ to the number $N$ and to each of these given $m_{A, M}(N)$ triangulations. If no new triangulation from $T_{M}(N)$ have appeared, then stop. Otherwise consider the list $L_{1}$ containing all new 
triangulations from $T_{M}(N)$ produced by $A$ and all $m_{A, M}(N)$ initial triangulations. Now we will proceed recursively. On every step we will apply the algorithm $A$ to every triangulation from the list of triangulations $L_{k-1}$ obtained on the previous step. If no new triangulations with $\leqq N$ simplices appeared as the result (i.e. if $\left.\bigcup_{T \in L_{k-1}} A(N, T) \subset L_{k-1}\right)$, then the algorithm stops. Otherwise we form a new list $L_{k}$ adding to the old list $L_{k-1}$ all new triangulations of $M$ with $\leqq N$ simplices produced by $A$. (In other words we define $L_{k}$ as $L_{k-1} \bigcup_{T \in L_{k-1}} A(N, T)$ ). ) Then the algorithm passes to the $(k+1)$-th step This algorithm must stop after a number of steps not exceeding the (finite) number of triangulations of $M$ with $\leqq N$ simplices. The definition of the poset $T_{A, M}(N)$ and the fact that we started from the list of representatives from all minimal elements of the poset guarantee that the described algorithm constructs the list $L_{M}(N)$ of all possible triangulations of $M$ with $\leqq N$ simplices

When the list $L_{M}(N)$ is known, one can check for any given abstract simplicial complex with $\leqq N$ simplices whether or not this complex is in $H_{M}$ just comparing this complex with all simplicial complexes from the list $L_{M}(N)$ and checking whether or not this complex is simplicially isomorphic to one of them. (Obviously, it is possible to check whether or not two given abstract simplicial complexes are simplicially isomorphic, cf [ABB], Proposition 216 .) Our definition of $A$ implies that the time of work of $A$ when it is applied to $N$ and to a triangulation from $T_{M}(N)$ is bounded from above by a recursive function of $N$ Hence, the time of work of the recognition algorithm described above is also bounded by a recursive function of $N$ Thus, for a certain recursive function $\lambda_{A}$ the problem of recognition whether or not a simplicial complex with $\leqq N$ simplices (of all dimensions) is a triangulation of $M$ has $\hat{\lambda}_{A}$-bounded Kolmogorov complexity not exceeding $C N \ln N m_{A, M}(N)$.

Now we can prove Theorem 11 by a simple juxtaposition of results of Lemma 31 and Lemma 32 Indeed, such a juxtaposition shows that for all sufficiently large $N$,

$$
m_{A, M}(N) \geqq \frac{C_{0}^{N}(n)}{C N \ln N}>C^{N}(n) .
$$

Here $C(n)$ is an arbitrary constant greater than one and less than $C_{0}(n)$ (for example, we can take $C(n)=\frac{1}{2}\left(1+C_{0}(n)\right)$ This proves Theorem 1.1 .

Now we are going to prove Theorem 15 . The conditions of Theorem $1.5 \mathrm{im}$ ply that for any triangulation $T \in T_{M}(\gamma(N))$ and for any triangulation $S$ of $M$ with $\geqq N$ simplices $F(S) \geqq F(T)$. Consider the algorithm $A_{\text {dist, } \theta \text {, finding for given }}$ $N, T \in T_{M}(N)$ all triangulations $V$ from $T_{M}(N)$ such that $\operatorname{dist}(T, V) \leqq \theta(N)$. Consider the partition of $T_{M}(N)$ into $A_{\text {dist }, 0 \text {-simple sets }}$ Let $D_{1}, \ldots, D_{l}$ denote those of these $A_{\text {dist }, 0}$-simple sets which have a non-empty intersection with $T_{M}(\gamma(N))$. Theorem 15 is an immediate corollary of the following two statements.

1) For every $i$ the minimum of the restriction of $F$ on $D_{i}$ is a $\theta$-distinctive local minimum of $F . T_{M} \rightarrow \mathbb{R}$.

2) For all sufficiently large $N$ the number $l$ of the sets $D_{i}$ is not less than $\left[C_{*}^{\gamma^{(N)}}(n)\right]$, where $C_{*}(n)>1$ is a constant depending only on $n$.

To prove 1) note that the definition of $D_{i}$ implies that if $T$ is a minimum of the restriction of $F$ on $D_{i}$ and a triangulation $S \notin D_{i}$ satisfies the inequality $\operatorname{dist}(S, T) \leqq O(N)$, then $S \notin T_{M}(N)$ Therefore the number of simplices of $S$ is 
greater than $N$. Since $D_{i}$ contains a triangulation $T_{i}$ from $T_{M}(\gamma(N)), F(T) \leqq$ $F\left(T_{i}\right) \leqq F(S)$. Hence, $F(S) \geqq F(T)$.

To prove 2) note that there exists an algorithm working in a time bounded by a recursive function of $N$ which for a given $N$ and $T \in T_{M}(\gamma(N))$ finds the set $D_{i}$ containing $T$. (It uses the algorithm finding the $\theta(N)$-ball in the metric dist around $T$ in $T_{M}(N)$ in the same fashion as the algorithm, described in the proof of Lemma 3.2 uses an algorithm $A$.) Thus, similarly to the proof of Lemma 3.2, we can conclude that a collection of representatives from sets $D_{i} \cap T_{M}(\gamma(N))$ for all $i \in\{1, \ldots, l\}$ is sufficient to construct $T_{M}(\gamma(N))$ and solve the recognition problem for all triangulations with $\leqq \gamma(N)$ simplices in a time bounded by a recursive function $\lambda_{1}$ of $\gamma(N)$. This argument yields an upper bound const $\gamma(N) \ln \gamma(N) l$ for $K_{R}^{\lambda_{1}}\left(H_{M}, \gamma(N)\right)$. The comparison with the lower bound provided by Lemma 3.1 implies the required lower bound for $l$ quite similarly to the proof of Theorem 1.1.

Acknowledgements The author would like to thank Professors B. Sturmfels, O. Viro and, especially, M. Gromov for fruitful discussions I would like to thank Prof. S. Weinberger for a very helpful discussion of the effective version of S Novikov's theorem. I am indebted to Dr Radel Ben-Av for explaining to me the connections between questions considered in this paper and Quantum Gravity. I would like to thank Prof. R Connelly for informing me about references $[\mathrm{Ca}, \mathrm{CH}]$.

A previous version of the present paper was distributed as a preprint entitled "Extremal triangulations of PL-manifolds."

\section{References}

[A] Alexander, J W : The combinatorial theory of complexes. Ann Math 31, 292-320 (1930)

[ABB] Acquistapace, F, Benedetti, R, Broglia, F.: Effectiveness-non-effectiveness in semialgebraic and PL geometry. Inv Math. 102 (1), 141-156 (1990)

[AM] Agishtein, M.E, Migdal, A A : Nucl. Phys. B 385, 395 (1992)

[AJK] Ambjorn, F.J , Jurkiewicz, J., Kristjansen, C F : Quantum gravity, dynamical triangulations and higher derivative regularization Nucl. Phys. B 393, 601 (1993)

[B] Barzdin, J M.: Complexity of programs to determine whether natural numbers not greater than $n$ belong to a recursively enumerable set. Soviet Math Doklady 9, 1251-1254 (1968)

[BHP] Boone, W.W , Haken, W., Poénaru, V.: On recursively unsolvable problems in topology and their classification In: "Contributions to Mathematical Logic," Arnold Schmidt, H, Schutte, K., Thiele, H -J. (eds.), Amsterdam; North Holland, 1968

[Ca] Cairns, S.S : Triangulated manifolds which are not Brower manifolds Ann Math. 41, $792-795$ (1940)

[C] Chaitin, G : Information, Randomness and Incompleteness. Singapore: World Scientific, 1987

[CMS] Cheeger, F J, Muller, W, Schrader, R.: On the curvature of piecewise-flat spaces. Commun Math. Phys. 92, 405-454 (1984)

[CH] Connelly, R, Henderson, D : A convex 3-complex not simplicially isomorphic to a strictly convex complex. Math. Proc Cambr. Phil. Soc 88, 299-306 (1980)

[D] Daley, R.P.: Minimal program complexity of sequences with restricted resources Inf and Control 23, 301-312 (1973)

[F] Fomenko, A.T : Differential Geometry and Topology N Y : Plenum, 1987

[G] Glaser, L.C.: Geometrical Combinatorial Topology, Vol. 1. Amsterdam: Van Nostrand, 1970

[Gr1] Gromov, M.: Hyperbolic manifolds, groups and actions Riemannian surfaces and related topics, Kra, I., Maskit, B. (ed.), Ann. of Math. Studies 97, 183-215 (1981) 
[Gr2] Gromov, M : Asymptotic invariants of infinite groups Geometric Group Theory, Niblo, G A, Roller, M A (ed ), Vol 2, London Math Soc Lecture Notes Series 182, 1993

[GV] Gross, M, Varsted, S : Nucl Phys B378, 367 (1992)

[H] Hopf, H: Fundamentalgruppe und Zweite Bettische Gruppe Comm Math Helv 14, 257-309 (1941/1942)

[J] Jurkiewicz, J : Simplicial gravity and random surfaces Nucl Phys B (Proc Suppl ) 30, 108-121 (1993)

[K] Kervaire, M : Smooth homology spheres and their fundamental groups Trans Amer Math Soc 149, 67-72 (1969)

[LV] Li, M, Vitanyi, P M B : Kolmogorov Complexity and its applications Handbook of Theoretical Computer Science, Jan van Leeuwen, (ed ), Elsevier, 1990, pp 187-254

[M] Yu I Manin: A course in Mathematical Logic Berlin-Heidelberg-New York Springer, 1977

[Mi] Miller, C F : Decision Problems for groups-Survey and reflections Algorithms and Classification in Combinatorial group theory, Baumslag, G, Miller, C F (eds ), BerlinHeidelberg-New York: Springer, 1989

[Mn] Milnor, J : Introduction to algebraic $K$-theory Annals of Mathematical Studies, Princeton, NJ: Princeton University Press, 1971

[NBA] Nabutovsky, A, Ben-Av, R : Non-computability arising in dynamical triangulation model of Four-Dimensional Quantum Gravity Commun in Math Phys 157, 93-98 (1993)

[N0] Nabutovsky, A : Non-recursive functions in real algebraic geometry Bull Amer Math Soc 20, 61-65 (1989)

[N1] Nabutovsky, A : Non-recursive functions, knots "with thick ropes" and self-clenching "thick" hyperspheres Comm in Pure and Appl Math 48, 381-428 (1995)

[N2] Nabutovsky, A : Disconnectedness of sublevel sets of some Riemannian functionals To appear in Geom Funct Anal

[N3] Nabutovsky, A : Fundamental group and contractible closed geodesics To appear in Comm on Pure and Appl Math

[N4] Nabutovsky, A : Einstein structures: Existence versus uniqueness Geom Funct Anal 5, 76-91 (1995)

[N5] Nabutovsky, A : Exponential growth of the number of connected components of sublevel sets of some Riemannian functionals In preparation

[P1] Pachner, U: Konstruktionsmethoden und das kombinatorische Homoomorphieproblem fur Triangulationen kompakter semilinearer Mannigfaltigkeiten Abh Math Sem Univ Hamburg 57, 69-85 (1986)

[P2] Pachner, U : P L Homeomorphic Manifolds are Equivalent by Elementary Shellings Europ J Combinatorics 12, 129-145 (1991)

[R] Rotman, J J : An introduction to the theory of groups Boston, MA: Allyn and Bacon, 1984

[S] Stanley, R: Subdivisions and local $h$-vectors J of A M S 5, 805-852 (1992)

[VKF] Volodin, I A, Kuznetsov, V E, Fomenko, A T : The problem of discriminating algorithmically the standard three-dimensional sphere Russ Math Surv 29, no 5, 71-172 (1974)

[ZL] Zvonkin, A K, Levin, L A : The complexity of finite objects and the development of the concepts of information and randomness by means of the theory of algorithms Russ Math Surv 25, no 6, 83-129 (1970) 